\title{
ON THE NUMBER OF REPRESENTATIONS OF INTEGERS BY SOME QUADRATIC FORMS IN TEN VARIABLES
}

\author{
G. LOMADZE
}

\begin{abstract}
A method of finding the so-called Liouville's type formulas for the number of representations of integers by

$a_{1}\left(x_{1}^{2}+x_{2}^{2}\right)+a_{2}\left(x_{3}^{2}+x_{4}^{2}\right)+a_{3}\left(x_{5}^{2}+x_{6}^{2}\right)+a_{4}\left(x_{7}^{2}+x_{8}^{2}\right)+a_{5}\left(x_{9}^{2}+x_{10}^{2}\right)$ quadratic forms is developed.
\end{abstract}

In the papers $[4,5]$ four classes of entire modular forms of weight 5 for the congruence subgroup $\Gamma_{0}(4 N)$ are constructed. The Fourier coefficients of these modular forms have a simple arithmetical sense. This allows one to get sometimes the so-called Liouville's type formulas for the number of representations of positive integers by positive quadratic forms in ten variables.

In the present paper we consider positive primitive quadratic forms

$$
\begin{aligned}
f & =a_{1}\left(x_{1}^{2}+x_{2}^{2}\right)+a_{2}\left(x_{3}^{2}+x_{4}^{2}\right)+a_{3}\left(x_{5}^{2}+x_{6}^{2}\right)+ \\
& +a_{4}\left(x_{7}^{2}+x_{8}^{2}\right)+a_{5}\left(x_{9}^{2}+x_{10}^{2}\right) .
\end{aligned}
$$

For the purpose of illustration we obtain a formula for the number of representations of positive integers by the form (1) for $a_{1}=\cdots=a_{4}=1 a_{5}=4$. In a similar way one can investigate as well other forms of the kind (1). As is well known, Liouville obtained in 1865 the corresponding formula for $a_{1}=\cdots=a_{5}=1$ only.

\section{SOME KNOWN RESUlts}

1.1. In this paper $N, a, d, k, n, q, r, s, \lambda$ denote positive integers; $b, u, v$ are odd positive integers; $p$ is a prime number; $\nu, l$ are non-negative integers; $H, c, g, h, j, m, x, y, \alpha, \beta, \gamma, \delta$ are integers; $i$ is an imaginary unit; $z, \tau$ are

1991 Mathematics Subject Classification. 11E25, 11E20.

Key words and phrases. Quadratic form, number of representations, entire modular form, congruence subgroup, Gaussian sum, Ramanujan's sum, theta-function, singular series. 
complex variables $(\operatorname{Im} \tau>0) ; e(z)=\exp 2 \pi i z ; Q=e(\tau) ;\left(\frac{h}{u}\right)$ is the generalized Jacobi symbol. Further, $\sum_{h \bmod q}$ and $\sum_{h \bmod q}^{\prime}$ denote respectively sums in which $h$ runs a complete and a reduced residue system modulo $q$.

Let

$$
\begin{gathered}
S(h, q)=\sum_{j \bmod q} e\left(\frac{h j^{2}}{q}\right) \quad \text { (Gaussian sum), } \\
c(h, q)=\sum_{j \bmod q}^{\prime} e\left(\frac{h j^{2}}{q}\right) \quad \text { (Ramanujan's sum), } \\
\vartheta_{g h}(z \mid \tau ; c, N)= \\
=\sum_{m \equiv c(\bmod N)}(-1)^{h(m-c) / N} e\left(\frac{1}{2 N}\left(m+\frac{g}{2}\right)^{2} \tau\right) e\left(\left(m+\frac{g}{2}\right) z\right)
\end{gathered}
$$

(theta-function with characteristics $g, h$ ),

hence

$$
\begin{gathered}
\frac{\partial^{n}}{\partial z^{n}} \vartheta_{g h}(z \mid \tau ; c, N)=(\pi i)^{n} \sum_{m \equiv c(\bmod N)}(-1)^{h(m-c) / N}(2 m+g)^{n} \times \\
\times e\left(\frac{1}{2 N}\left(m+\frac{g}{2}\right)^{2} \tau\right) e\left(\left(m+\frac{g}{2}\right) z\right) .
\end{gathered}
$$

Put

$$
\begin{gathered}
\vartheta_{g h}(\tau ; c, N)=\vartheta_{g h}(0 \mid \tau ; c, N), \\
\vartheta_{g h}^{(n)}(\tau ; c, N)=\left.\frac{\partial^{n}}{\partial z^{n}} \vartheta_{g h}(z \mid \tau ; c, N)\right|_{z=0} .
\end{gathered}
$$

It is known (see, for example, [3], p. 112, formulas (2.3) and (2.5)) that

$$
\begin{aligned}
\vartheta_{g+2 j, h}(\tau ; c, N) & =\vartheta_{g h}(\tau ; c+j, N), \\
\vartheta_{g+2 j, h}^{(n)}(\tau ; c, N) & =\vartheta_{g h}^{(n)}(\tau ; c+j, N), \\
\vartheta_{g h}\left(\tau ; c+N_{j}, N\right) & =(-1)^{h j} \vartheta_{g h}(\tau ; c, N), \\
\vartheta_{g h}^{(n)}\left(\tau ; c+N_{j}, N\right) & =(-1)^{h j} \vartheta_{g h}^{(n)}(\tau ; c, N) .
\end{aligned}
$$

From (1.3), in particular, according to the notations (1.5), it follows that

$$
\begin{gathered}
\vartheta_{g h}(\tau ; 0, N)=\sum_{m=-\infty}^{\infty}(-1)^{h m} Q^{(2 N m+g)^{2} / 8 N}, \\
\vartheta_{g h}^{(n)}(\tau ; 0, N)=(\pi i)^{n} \sum_{m=-\infty}^{\infty}(-1)^{h m}(2 N m+g)^{n} Q^{(2 N m+g)^{2} / 8 N} .
\end{gathered}
$$


From (1.8) and (1.9) it follows that

$$
\vartheta_{-g, h}(\tau ; 0, N)=\vartheta_{g h}(\tau ; 0, N), \quad \vartheta_{-g, h}^{(n)}(\tau ; 0, N)=(-1)^{n} \vartheta_{g h}^{(n)}(\tau ; 0, N) .
$$

Everywhere in this paper $a$ denote a least common multiple of the coefficients $a_{k}$ of the quadratic form (1) and $\Delta=\prod_{k=1}^{5} a_{k}^{2}$ is its determinant.

Denoting by $r(n ; f)$ the number of representations of $n$ by the form (1), we get

$$
\prod_{k=1}^{5} \vartheta_{00}^{2}\left(\tau ; 0, a_{k}\right)=1+\sum_{n=1}^{\infty} r(n ; f) Q^{n} .
$$

Further, put

$$
\theta(\tau ; f)=1+\sum_{n=1}^{\infty} \rho(n ; f) Q^{n}
$$

where

$$
\rho(n ; f)=\frac{\pi^{5}}{4 ! \Delta^{1 / 2}} n^{4} \sum_{q=1}^{\infty} A(q)
$$

(singular series of the problem) and

$$
A(q)=q^{-10} \sum_{h \bmod q}^{\prime} e\left(-\frac{h n}{q}\right) \prod_{k=1}^{5} S^{2}\left(a_{k} h, q\right) .
$$

Finally let

$$
\Gamma_{0}(4 N)=\left\{\frac{\alpha \tau+\beta}{\gamma \tau+\delta} \mid \alpha \delta-\beta \gamma=1, \gamma \equiv 0(\bmod 4 N)\right\}
$$

(nonhomogeneous congruence subgroup).

1.2. For the convenience of references we quote some known results as the following lemmas.

Lemma 1. If $(h, q)=1$, then

$$
S(k h, k q)=k S(h, q) .
$$

Lemma 2 (see, for example, [6], p. 13, Lemma 6). If $(h, q)=1$, then

$$
\begin{aligned}
S^{2}(h, q) & =\left(\frac{-1}{q}\right) q \text { for } q \equiv 1(\bmod 2), \\
& =2 i^{h} q \text { for } q \equiv 0(\bmod 4) \\
& =0 \quad \text { for } q \equiv 2(\bmod 4)
\end{aligned}
$$


Lemma 3 (see, for example, [6], p. 16, Lemma 8). If $(h, q)=1$, then

$$
S(h, u)=\left(\frac{h}{u}\right) i^{(u-1)^{2} / 4} u^{1 / 2} .
$$

Lemma 4 (see, for example $[6]$, p. 177, formula 20). Let $q=p^{\lambda}$ and $p^{\nu} \| h$. Then

$$
\begin{aligned}
c(h, q) & =0 \text { for } \nu<\lambda-1, \\
& =-p^{\lambda-1} \text { for } \quad \nu=\lambda-1, \\
& =p^{\lambda-1}(p-1) \text { for } \quad \nu>\lambda-1 .
\end{aligned}
$$

Lemma 5 (see, for example, [2], p. 14, Lemma 10). Let

$$
\left.\chi_{p}=1+A(p)+A(p)^{2}\right)+\cdots .
$$

Then

$$
\sum_{q=1}^{\infty} A(q)=\prod_{p} \chi_{p} .
$$

Lemma 6 ([1], pp. 811 and 953). The entire modular form $F(\tau)$ of weight $r$ for the congruence subgroup $\Gamma_{0}(4 N)$ is identically zero, if in its expansion in the series

$$
\begin{gathered}
F(\tau)=\sum_{m=0}^{\infty} C_{m} Q^{m} \\
C_{m}=0 \text { for all } m \leq \frac{r}{3} N \prod_{p \mid 4 N}\left(1+\frac{1}{p}\right) .
\end{gathered}
$$

Lemma 7 ([7], p. 18, Lemma 14). The function $\theta(\tau ; f)$ is an entire modular form of weight 5 and character $\chi(\delta)=\operatorname{sgn} \delta\left(\frac{-\Delta}{|\delta|}\right)$ for $\Gamma_{0}(4 a)$.

Lemma 8 ([2], p. 21, the remark to Lemma 18). The function $\prod_{k=1}^{5} \vartheta_{00}\left(\tau ; 2, a_{k}\right)$ is an entire modular form of weight 5 and character $\chi(\delta)=\operatorname{sgn} \delta\left(\frac{-\Delta}{|\delta|}\right)$ for $\Gamma_{0}(4 a)$.

Lemma 9 ([4], p. 67, Theorem $1^{1}$ and [5], p. 193, Theorem 1). For a given $N$ the functions

(1) $\Psi_{2}\left(\tau ; g_{1}, g_{2} ; h_{1}, h_{2} ; 0,0 ; N_{1}, N_{2}\right)=$

$$
\begin{aligned}
& =\frac{1}{N_{1}^{2}} \vartheta_{g_{1} h_{1}}^{(4)}\left(\tau ; 0,2 N_{1}\right) \vartheta_{g_{2} h_{2}}\left(\tau ; 0,2 N_{2}\right)+ \\
& +\frac{1}{N_{2}^{2}} \vartheta_{g_{1} h_{1}}\left(\tau ; 0,2 N_{1}\right) \vartheta_{g_{2} h_{2}}^{(4)}\left(\tau ; 0,2 N_{2}\right)- \\
& -\frac{6}{N_{1} N_{2}} \vartheta_{g_{1} h_{1}}^{\prime \prime}\left(\tau ; 0,2 N_{1}\right) \vartheta_{g_{2} h_{2}}^{\prime \prime}\left(\tau ; 0,2 N_{2}\right),
\end{aligned}
$$

\footnotetext{
${ }^{1}$ There is a misprint in the formulation of Theorem 1 [4, p. 67] which can be easily corrected by substituting 5 for 10 and vice versa.
} 
where

(a) $2\left|g_{1}, 2\right| g_{2}, N_{1}\left|N, N_{2}\right| N, 4\left|N\left(\frac{h_{1}^{2}}{N_{1}}+\frac{h_{2}^{2}}{N_{2}}\right), 4\right| \frac{g_{1}^{2}}{4 N_{1}}+\frac{g_{2}^{2}}{4 N_{2}}$,

(b) for all $\alpha$ and $\delta$ with $\alpha \delta \equiv 1(\bmod 4 N)$

$$
\begin{gathered}
\left(\frac{N_{1} N_{2}}{|\delta|}\right) \Psi_{2}\left(\tau ; \alpha g_{1}, \alpha g_{2} ; h_{1}, h_{2} ; 0,0 ; N_{1}, N_{2}\right)= \\
=\left(\frac{\Delta}{|\delta|}\right) \Psi_{2}\left(\tau ; g_{1}, g_{2} ; h_{1}, h_{2} ; 0,0 ; N_{1}, N_{2}\right),
\end{gathered}
$$

(2) $\Psi_{3}\left(\tau ; g_{1}, \ldots, g_{4} ; h_{1}, \ldots, h_{4} ; 0, \ldots, 0 ; N_{1}, \ldots, N_{4}\right)=$

$$
\begin{aligned}
& =\left\{\frac{1}{N_{1}} \vartheta_{g_{1} h_{1}}^{\prime \prime}\left(\tau ; 0,2 N_{1}\right) \vartheta_{g_{2} h_{2}}\left(\tau ; 0,2 N_{2}\right)-\right. \\
& \left.-\frac{1}{N_{2}} \vartheta_{g_{1} h_{1}}\left(\tau ; 0,2 N_{1}\right) \vartheta_{g_{2} h_{2}}^{\prime \prime}\left(\tau ; 0,2 N_{2}\right)\right\} \times \\
& \times \vartheta_{g_{3} h_{3}}^{\prime}\left(\tau ; 0,2 N_{3}\right) \vartheta_{g_{4} h_{4}}\left(\tau ; 0,2 N_{4}\right)
\end{aligned}
$$

and

(3) $\Psi_{4}\left(\tau ; g_{1}, \ldots, g_{4} ; h_{1}, \ldots, h_{4} ; 0, \ldots, 0 ; N_{1}, \ldots, N_{4}\right)=$ $=\prod_{k=1}^{3} \vartheta_{g_{k} h_{k}}^{\prime}\left(\tau ; 0,2 N_{k}\right) \vartheta_{g_{4} h_{4}}\left(\tau ; 0,2 N_{4}\right)$,

where

(a) $2\left|g_{k}, N_{k}\right| N(k=1,2,3,4), 4\left|N \sum_{k=1}^{4} \frac{h_{k}^{2}}{N_{k}}, 4\right| \sum_{k=1}^{4} \frac{g_{k}^{2}}{4 N_{k}}$,

(b) for all $\alpha$ and $\delta$ with $\alpha \delta \equiv 1(\bmod 4 N)$

$$
\begin{aligned}
& \left(\frac{\prod_{k=1}^{4} N_{k}}{|\delta|}\right) \Psi_{j}\left(\tau ; \alpha g_{1}, \ldots, \alpha g_{4} ; h_{1}, \ldots, h_{4} ; 0, \ldots, 0 ; N_{1}, \ldots, N_{4}\right)= \\
= & \operatorname{sgn} \delta\left(\frac{-\Delta}{|\delta|} \Psi_{j}\left(\tau ; g_{1}, \ldots, g_{4} ; h_{1}, \ldots, h_{4} ; 0, \ldots, 0 ; N_{1}, \ldots, N_{4}\right) \quad(j=3,4),\right.
\end{aligned}
$$

are entire modular forms of weight 5 and character $\chi(\delta)=\operatorname{sgn} \delta\left(\frac{-\Delta}{|\delta|}\right)$ for $\Gamma_{0}(4 N)$.

\section{Summation of the Singular Series $\rho(n ; f)$}

Everywhere in this section $\alpha, \beta, \gamma$ denote non-negative integers and $m$ positive odd integers.

Lemma 10. Let $n=2^{\alpha} m, a_{k}=2^{\gamma_{k}} b_{k}(k=1,2, \ldots, 5),\left(b_{1}, \ldots, b_{5}\right)=1$, $b=\left[b_{1}, \ldots, b_{5}\right], \gamma_{5} \geq \gamma_{4} \geq \gamma_{3} \geq \gamma_{2} \geq \gamma_{1}=0, \gamma=\sum_{k=1}^{5} \gamma_{k}$. Then

$$
\begin{aligned}
& \chi_{2}=1+(-1)^{\left(b_{1}-m\right) / 2} \text { for } 0 \leq \alpha \leq \gamma_{2}-2, \\
& =1 \text { for } \alpha=\gamma_{2}-1, \alpha=\gamma_{2}<\gamma_{3}, \gamma_{2}=\gamma_{3} \leq \alpha=\gamma_{4}-1, \\
& \qquad \gamma_{2}+1=\gamma_{3} \leq \alpha=\gamma_{4}-1, \gamma_{2}=\gamma_{3} \leq \alpha=\gamma_{4}<\gamma_{5},
\end{aligned}
$$




$$
\begin{aligned}
& \gamma_{2}+1=\gamma_{3} \leq \alpha=\gamma_{4}<\gamma_{5} \\
& =1+(-1)^{\left(b_{1}+b_{2}\right) / 2}\left(1-2^{\gamma_{2}-\alpha} \cdot 3\right) \text { for } \gamma_{2}+1 \leq \alpha<\gamma_{3} \text {, } \\
& =1+(-1)\left(\sum_{k=1}^{3} b_{k}-m\right) / 2 \cdot 2^{\gamma_{2}+\gamma_{3}-2 \alpha-2} \text { for } \gamma_{2}=\gamma_{3} \leq \alpha<\gamma_{4}-2 \text {, } \\
& \gamma_{2}+1=\gamma_{3} \leq \alpha \leq \gamma_{4}-2 \\
& =1+(-1)^{\left(b_{1}+b_{2}\right) / 2}\left(1-2^{\gamma_{2}-\gamma_{3}+1}\right)+(-1)\left(\sum_{k=1}^{3} b_{k}-m\right) / 2 \cdot 2^{\gamma_{2}-\gamma_{3}-2 \alpha-2} \\
& \text { for } \gamma_{2}+2 \leq \gamma_{3} \leq \alpha \leq \gamma_{4}-2 \text {, } \\
& =1+(-1)^{\left(b_{1}+b_{2}\right) / 2}\left(1-2^{\gamma_{2}-\gamma_{3}+1}\right) \text { for } \gamma_{2}+2 \leq \gamma_{3} \leq \alpha=\gamma_{4}-1 \text {, } \\
& \gamma_{2}+2 \leq \gamma_{3} \leq \alpha=\gamma_{4}<\gamma_{5} \\
& =1+(-1)^{\left(b_{1}+b_{2}\right) / 2}\left(1-2^{\gamma_{2}-\gamma_{3}+1}\right)+(-1)\left(\sum_{k=1}^{4} b_{k}\right) / 2 \times \\
& \times 2^{\gamma_{2}+\gamma_{3}-2 \gamma_{4}}\left\{\frac{1}{7}\left(1-2^{-3\left(\alpha-\gamma_{4}-1\right)}\right)-2^{-3\left(\alpha-\gamma_{4}\right)}\right\} \\
& \text { for } \gamma_{4}+1 \leq \alpha<\gamma_{5} \text { but } \gamma_{4} \geq \gamma_{3} \geq \gamma_{2}+2 \text {, } \\
& =1+(-1)\left(\sum_{k=1}^{4} b_{k}\right) / 2 \cdot 2^{\gamma_{2}+\gamma_{3}-2 \gamma_{4}}\left\{\frac{1}{7}\left(1-2^{-3\left(\alpha-\gamma_{4}-1\right)}\right)-2^{-3\left(\alpha-\gamma_{4}\right)}\right\} \\
& \text { for } \gamma_{4}+1 \leq \alpha<\gamma_{5} \text { but } \gamma_{4} \geq \gamma_{3}=\gamma_{2} \text { or } \gamma_{4} \geq \gamma_{3}=\gamma_{2}+1 \text {, } \\
& =1+(-1)^{\left(\sum_{k=1}^{5} b_{k}-m\right) / 2} \cdot 2^{\gamma-4 \alpha-4} \text { for } \alpha \geq \gamma_{5}=\gamma_{4} \geq \gamma_{3}=\gamma_{2} \text {, } \\
& \alpha \geq \gamma_{5}=\gamma_{4} \geq \gamma_{3}=\gamma_{2}+1 \\
& =1+(-1)^{\left(b_{1}+b_{2}\right) / 2}\left(1-2^{\gamma_{2}-\gamma_{3}+1}\right)+(-1)\left(\sum_{k=1}^{5} b_{k}-m\right) / 2 \cdot 2^{\gamma-4 \alpha-4} \\
& \text { for } \alpha \geq \gamma_{5}=\gamma_{4} \geq \gamma_{3} \geq \gamma_{2}+2 \\
& =1+(-1)^{\left(b_{1}+b_{2}\right) / 2}\left(1-2^{\gamma_{2}-\gamma_{3}+1}\right)+(-1)\left(\sum_{k=1}^{4} b_{k}\right) / 2 \times \\
& \times 2^{\gamma_{2}+\gamma_{3}-2 \gamma_{4}}\left(1-2^{-3\left(\gamma_{5}-\gamma_{4}-1\right)}\right) / 7+(-1)\left(\sum_{k=1}^{5} b_{k}-m\right) / 2 \cdot 2^{\gamma-4 \alpha-4} \\
& \text { for } \alpha \geq \gamma_{5}>\gamma_{4} \geq \gamma_{3} \geq \gamma_{2}+2 \text {, } \\
& =1+(-1)\left(\sum_{k=1}^{4} b_{k}\right) / 2 \cdot 2^{\gamma_{2}+\gamma_{3}-2 \gamma_{4}}\left(1-2^{-3\left(\gamma_{5}-\gamma_{4}-1\right)}\right) / 7+ \\
& +(-1)^{\left(\sum_{k=1}^{5} b_{k}-m\right) / 2} \cdot 2^{\gamma-4 \alpha-4} \text { for } \alpha \geq \gamma_{5}>\gamma_{4} \geq \gamma_{3}=\gamma_{2} \text {, } \\
& \alpha \geq \gamma_{5}>\gamma_{4} \geq \gamma_{3}=\gamma_{2}+1
\end{aligned}
$$

Proof. I. If in (1.14) we put $q=2^{\lambda}$ and then instead of $h$ introduce a new letter of summation $y$ defined by the congruence $h \equiv b y\left(\bmod 2^{\lambda}\right)$, then we get

$$
A\left(2^{\lambda}\right)=2^{-10 \lambda} \sum_{y \bmod 2^{\lambda}}^{\prime} e\left(-2^{\alpha-\lambda} m b y\right) \prod_{k=1}^{5} S^{2}\left(2^{\gamma_{k}} b_{k} b y, 2^{\lambda}\right)
$$


From (2.1), according to Lemmas 1, 2, and 4 it follows that

(1) for $\lambda=1, \gamma_{k}+1(k=2,3,4,5)$

$$
A\left(2^{\lambda}\right)=0
$$

because $S^{2}\left(b_{1} b y, 2\right)=0, S^{2}\left(2^{\gamma_{k}} b_{k} b y, 2^{\gamma_{k}+1}\right)=0(k=2,3,4,5)$;

(2) for $2 \leq \lambda \leq \gamma_{2}$

$$
\begin{aligned}
A\left(2^{\lambda}\right) & =2^{-10 \lambda} \sum_{y \bmod 2^{\lambda}}^{\prime} e\left(-2^{\alpha-\lambda} m b y\right)\left(2 i^{b_{i} b y} \cdot 2^{\lambda}\right) 2^{8 \lambda}= \\
& =2^{1-\lambda} \sum_{y \bmod 2^{\lambda}}^{\prime} e\left(\frac{b_{1} b y}{4}-\frac{2^{\alpha} m b y}{2^{\lambda}}\right)= \\
& =2^{1-\lambda} e\left(\frac{\left(b_{1}-2^{\alpha-\lambda+2} m\right) b}{4}\right) \sum_{y=0}^{2^{\lambda-1}-1} e\left(\frac{\left(2^{\lambda-2} b_{1}-2^{\alpha} m\right) b y}{2^{\lambda-1}}\right)= \\
& = \begin{cases}e\left(\left(b_{1}-2^{\alpha-\lambda+2} m\right) b / 4\right) & \text { if } 2^{\lambda-1} \mid\left(2^{\lambda-2} b_{1}-2^{\alpha} m\right) b, \\
0 & \text { if } 2^{\lambda-1} \dagger\left(2^{\lambda-2} b_{1}-2^{\alpha} m\right) b,\end{cases}
\end{aligned}
$$

i.e.,

$$
\begin{aligned}
A\left(2^{\lambda}\right) & =(-1)^{\left.b_{1}-m\right) / 2} \text { if } \lambda=\alpha+2 \\
& =0 \text { if } \lambda \neq \alpha+2
\end{aligned}
$$

(3) for $\gamma_{2}+2 \leq \lambda \leq \gamma_{3}$

$$
\begin{aligned}
A\left(2^{\lambda}\right) & =2^{-10 \lambda} \sum_{y \bmod 2^{\lambda}}^{\prime} e\left(-2^{\alpha-\lambda} m b y\right)\left(2 i^{b_{i} b y} \cdot 2^{\lambda}\right) 2^{2 \gamma_{2}} \cdot 2 i^{b_{2} b y} \times \\
& \times 2^{\lambda-\gamma_{2}} \cdot 2^{6 \lambda}=2^{\gamma_{2}-2 \lambda+2}(-1)^{\left(b_{1}+b_{2}\right) / 2} c\left(2^{\alpha} m b, 2^{\lambda}\right),
\end{aligned}
$$

hence

$$
\begin{aligned}
A\left(2^{\lambda}\right) & =(-1)^{\left(b_{2}+b_{2}\right) / 2} \cdot 2^{\gamma_{2}+1-\lambda} \text { if } \lambda<\alpha+1, \\
& =-(-1)^{\left(b_{2}+b_{2}\right) / 2} \cdot 2^{\gamma_{2}-\alpha} \text { if } \lambda=\alpha+1, \\
& =0 \text { if } \lambda>\alpha+1
\end{aligned}
$$

(4) for $\gamma_{3}+2 \leq \lambda \leq \gamma_{4}$

$$
\begin{aligned}
A\left(2^{\lambda}\right) & =2^{-10 \lambda} \sum_{y \bmod 2^{\lambda}}^{\prime} e\left(-2^{\alpha-\lambda} m b y\right)\left(2 i^{b_{1} b y} \cdot 2^{\lambda}\right) 2^{2 \gamma_{2}} \times \\
& \times\left(2 i^{b_{2} b y} \cdot 2^{\lambda-\gamma_{2}}\right) 2^{2 \gamma_{3}}\left(2 i^{b_{3} b y} \cdot 2^{\lambda-\gamma_{3}}\right) \cdot 2^{4 \lambda}=
\end{aligned}
$$


498

$$
\begin{aligned}
& =2^{\gamma_{2}+\gamma_{3}-3 \lambda+3} \sum_{y \bmod 2^{\lambda}}^{\prime} e\left(\frac{\left(b_{1}+b_{2}+b_{3}\right) b y}{4}-\frac{2^{\alpha} m b y}{2^{\lambda}}\right)= \\
& =2^{\gamma_{2}+\gamma_{3}-3 \lambda+3} e\left(\frac{1}{4}\left(\left(b_{1}+b_{2}+b_{3}\right) b-2^{\alpha-\lambda+2} m b\right)\right) \times \\
& \times \sum_{y=0}^{2^{\lambda-1}-1} e\left(2^{1-\lambda}\left(2^{\lambda-2}\left(b_{1}+b_{2}+b_{3}\right)-2^{\alpha} m\right) b y\right),
\end{aligned}
$$

hence, as in case (2)

$$
\begin{aligned}
& A\left(2^{\lambda}\right)=(-1)\left(\sum_{k=1}^{3} b_{k}-m\right) / 2 \cdot 2^{\gamma_{2}+\gamma_{3}-2 \alpha-2} \text { if } \lambda=\alpha+2, \\
& =0 \text { if } \lambda \neq \alpha+2 \text {; }
\end{aligned}
$$

(5) for $\gamma_{4}+2 \leq \lambda \leq \gamma_{5}$

$$
\begin{aligned}
& A\left(2^{\lambda}\right)=2^{-10 \lambda} \sum_{y \bmod 2^{\lambda}}^{\prime} e\left(-2^{\alpha-\lambda} m b y\right)\left(2 i^{b_{1} b y} \cdot 2^{\lambda}\right)\left(2^{2 \gamma_{2}} \cdot 2 i^{b_{2} b y} \cdot 2^{\lambda-\gamma_{2}}\right) \times \\
& \times\left(2^{2 \gamma_{3}} \cdot 2 i^{b_{3} b y} \cdot 2^{\lambda-\gamma_{3}}\right)\left(2^{2 \gamma_{4}} \cdot 2 i^{b_{4} b y} \cdot 2^{\lambda-\gamma_{4}}\right) 2^{2 \lambda}= \\
& =2^{\gamma_{2}+\gamma_{3}+\gamma_{4}-4 \lambda+4}(-1)\left(\sum_{k=1}^{4} b_{k}\right) / 2 c\left(2^{\alpha} b m, 2^{\lambda}\right) \text {, }
\end{aligned}
$$

hence

$$
\begin{aligned}
A\left(2^{\lambda}\right) & =(-1)\left(\sum_{k=1}^{4} b_{k}\right) / 2 \cdot 2^{\gamma-\gamma_{5}-3 \lambda-3} \text { if } \lambda<\alpha+1, \\
& =-(-1)\left(\sum_{k=1}^{4} b_{k}\right) / 2 \cdot 2^{\gamma-\gamma_{5}-3 \alpha} \text { if } \lambda=\alpha+1, \\
& =0 \text { if } \lambda>\alpha+1
\end{aligned}
$$

(6) for $\lambda>\gamma_{5}+1$

$$
\begin{aligned}
A\left(2^{\lambda}\right) & =2^{-10 \lambda} \sum_{y \bmod 2^{\lambda}}^{\prime} e\left(-2^{\alpha-\lambda} m b y\right)\left(2 i^{b_{1} b y} \cdot 2^{\lambda}\right) 2^{2 \gamma_{2}} \times \\
& \times\left(2 i^{b_{2} b y} \cdot 2^{\lambda-\gamma_{2}}\right) 2^{2 \gamma_{3}}\left(2 i^{b_{3} b y} \cdot 2^{\lambda-\gamma_{3}}\right) \times \\
& \times\left(2^{2 \gamma_{4}} \cdot 2 i^{b_{4} b y} \cdot 2^{\lambda-\gamma_{4}}\right)\left(2^{2 \gamma_{5}} \cdot 2 i^{b_{5} b y} \cdot 2^{\lambda-\gamma_{5}}\right)= \\
& =2^{\gamma-5 \lambda+5} \sum_{y \bmod 2^{\lambda}}^{\prime} e\left(\frac{\sum_{k=1}^{5} b_{k} b y}{4}-\frac{2^{\alpha} m b y}{2^{\lambda}}\right)= \\
& =2^{\gamma-5 \lambda+5} e\left(\frac{1}{4}\left(\sum_{k=1}^{5} b_{k} b-2^{\alpha-\lambda+2} m b\right)\right) \times
\end{aligned}
$$




$$
\times \sum_{y=0}^{2^{\lambda-1}-1} e\left(2^{1-\lambda}\left(2^{\lambda-2} \sum_{k=1}^{5} b_{k}-2^{\alpha} m\right) b y\right),
$$

hence, as in case (2),

$$
\begin{aligned}
A\left(2^{\lambda}\right) & =(-1)\left(\sum_{k=1}^{5} b_{k}-m\right) / 2 \cdot 2^{\gamma-4 \alpha-4} \text { if } \lambda=\alpha+2, \\
& =0 \text { if } \lambda \neq \alpha+2
\end{aligned}
$$

II. According to (1.15) and (2.2), we have

$$
\begin{aligned}
\chi_{2} & =1+\sum_{\lambda=2}^{\gamma_{2}} A\left(2^{\lambda}\right)+\sum_{\lambda=\gamma_{2}+2}^{\gamma_{3}} A\left(2^{\lambda}\right)+\sum_{\lambda=\gamma_{3}+2}^{\gamma_{4}} A\left(2^{\lambda}\right)+ \\
& +\sum_{\lambda=\gamma_{4}+2}^{\gamma_{5}} A\left(2^{\lambda}\right)+\sum_{\lambda=\gamma_{5}+2}^{\infty} A\left(2^{\lambda}\right) .
\end{aligned}
$$

Consider the following cases:

(1) Let $0 \leq \alpha \leq \gamma_{2}-2$. Then from (2.8), (2.3), (2.31), (2.42), (2.5 $)$, $\left(2.6_{2}\right)$, and $\left(2.7_{1}\right)$ we get

$$
\chi_{2}=1+\sum_{\lambda=2}^{\gamma_{2}} A\left(2^{\lambda}\right)=1+(-1)^{\left(b_{1}-m\right) / 2} .
$$

(2) Let $\alpha=\gamma_{2}-1$ or $\alpha=\gamma_{2}<\gamma_{3}$. Then from (2.8), (2.31), (2.4 $),\left(2.5_{1}\right)$, $\left(2.6_{2}\right)$, and $\left(2.7_{1}\right)$ we get

$$
\chi_{2}=1 .
$$

(3) Let $\gamma_{2}+1 \leq \alpha<\gamma_{3}$. Then from (2.8), (2.31), (2.4), (2.41), (2.4 $)$, $\left(2.5_{1}\right),\left(2.6_{2}\right)$, and $\left(2.7_{1}\right)$ we get

$$
\chi_{2}=1+\sum_{\lambda=\gamma_{2}+2}^{\gamma_{3}} A\left(2^{\lambda}\right)=1+\sum_{\lambda=\gamma_{2}+2}^{\alpha}(-1)^{\left(b_{1}+b_{2}\right) / 2} \cdot 2^{\gamma_{2}+1-\lambda}-(-1)^{\left(b_{1}+b_{2}\right) / 2} \cdot 2^{\gamma_{2}-\alpha}
$$

(4) Let $\gamma_{2}=\gamma_{3} \leq \alpha \leq \gamma_{4}-2$ or $\gamma_{2}+1=\gamma_{3} \leq \alpha \leq \gamma_{4}-2$. Then from (2.8), $\left(2.3_{1}\right),(2.5),\left(2.5_{1}\right),\left(2.6_{2}\right)$, and $\left(2.7_{1}\right)$ we get

$$
\chi_{2}=1+\sum_{\lambda=\gamma_{3}+2}^{\gamma_{4}} A\left(2^{\lambda}\right)=1+(-1)^{\left(\sum_{k=1}^{3} b_{k}-m\right) / 2} \cdot 2^{\gamma_{2}+\gamma_{3}-2 \alpha-2} .
$$


(5) Let $\gamma_{2}+2 \leq \gamma_{3} \leq \alpha \leq \gamma_{4}-2$. Then from (2.8), (2.31), (2.4), (2.5), $\left(2.5_{1}\right),\left(2.6_{2}\right)$, and $\left(2.7_{1}\right)$ we get

$$
\begin{gathered}
\chi_{2}=1+\sum_{\lambda=\gamma_{2}+2}^{\gamma_{3}} A\left(2^{\lambda}\right)+\sum_{\lambda=\gamma_{3}+2}^{\gamma_{4}} A\left(2^{\lambda}\right)= \\
=1+\sum_{\lambda=\gamma_{2}+2}^{\gamma_{3}}(-1)^{\left(b_{1}+b_{2}\right) / 2} \cdot 2^{\gamma_{2}+1-\lambda}+(-1)^{\left(\sum_{k=1}^{3} b_{k}-m\right) / 2} \cdot 2^{\gamma_{2}+\gamma_{3}-2 \alpha-2} .
\end{gathered}
$$

(6) Let $\gamma_{2}=\gamma_{3} \leq \alpha=\gamma_{4}-1$ or $\gamma_{2}+1=\gamma_{3} \leq \alpha=\gamma_{4}-1$ or $\gamma_{2}=\gamma_{3} \leq$ $\alpha=\gamma_{4}<\gamma_{5}$ or $\gamma_{2}+1=\gamma_{3} \leq \alpha=\gamma_{4}<\gamma_{5}$. Then from (2.8), (2.3 $),\left(2.5_{1}\right)$, $\left(2.6_{2}\right)$, and $\left(2.7_{1}\right)$ we get

$$
\chi_{2}=1 .
$$

(7) Let $\gamma_{2}+2 \leq \gamma_{3} \leq \alpha=\gamma_{4}-1$ or $\gamma_{2}+2 \leq \gamma_{3} \leq \alpha=\gamma_{4}<\gamma_{5}$. Then from $(2.8),\left(2.3_{1}\right),(2.4),\left(2.4_{2}\right),\left(2.5_{1}\right),\left(2.6_{1}\right)$, and $\left(2.7_{1}\right)$ we get

$$
\chi_{2}=1+\sum_{\lambda=\gamma_{2}+2}^{\gamma_{3}} A\left(2^{\lambda}\right)=1+\sum_{\lambda=\gamma_{2}+2}^{\gamma_{3}}(-1)^{\left(b_{1}+b_{2}\right) / 2} \cdot 2^{\gamma_{2}+1-\lambda} .
$$

8) Let $\gamma_{4}+1 \leq \alpha<\gamma_{5}$, but $\gamma_{4} \geq \gamma_{3} \geq \gamma_{2}+2$. Then from (2.8), (2.3 $)$, (2.4), $\left(2.5_{1}\right),(2.6),\left(2.6_{1}\right)$, and $\left(2.7_{1}\right)$ we get

$$
\begin{gathered}
\chi_{2}=1+\sum_{\lambda=\gamma_{2}+2}^{\gamma_{3}} A\left(2^{\lambda}\right)+\sum_{\lambda=\gamma_{4}+2}^{\gamma_{5}} A\left(2^{\lambda}\right)= \\
=1+\sum_{\lambda=\gamma_{2}+2}^{\gamma_{3}}(-1)^{\left(b_{1}+b_{2}\right) / 2} \cdot 2^{\gamma_{2}+1-\lambda}+ \\
+\sum_{\lambda=\gamma_{4}+2}^{\alpha}(-1)\left(\sum_{k=1}^{4} b_{k}\right) / 2 \cdot 2^{\gamma-\gamma_{5}-3 \lambda+3}-(-1)\left(\sum_{k=1}^{4} b_{k}\right) / 2 \cdot 2^{\gamma-\gamma_{5}-3 \alpha} .
\end{gathered}
$$

(9) Let $\gamma_{4}+1 \leq \alpha<\gamma_{5}$, but $\gamma_{4} \geq \gamma_{3}=\gamma_{2}$ or $\gamma_{4} \geq \gamma_{3}=\gamma_{2}+1$. Then from $(2.8),\left(2.3_{1}\right),\left(2.5_{1}\right),(2.6),\left(2.6_{1}\right)$, and $\left(2.7_{1}\right)$ we get

$$
\begin{gathered}
\chi_{2}=1+\sum_{\lambda=\gamma_{4}+2}^{\gamma_{5}} A\left(2^{\lambda}\right)+\sum_{\lambda=\gamma_{5}+2}^{\infty} A\left(2^{\lambda}\right)= \\
=1+\sum_{\lambda=\gamma_{4}+2}^{\alpha}(-1)\left(\sum_{k=1}^{4} b_{k}\right) / 2 \cdot 2^{\gamma-\gamma_{5}-3 \lambda+3}-(-1)\left(\sum_{k=1}^{4} b_{k}\right) / 2 \cdot 2^{\gamma-\gamma_{5}-3 \alpha} .
\end{gathered}
$$


(10) Let $\alpha \geq \gamma_{5}=\gamma_{4} \geq \gamma_{3}=\gamma_{2}$ or $\alpha \geq \gamma_{5}=\gamma_{4} \geq \gamma_{3}=\gamma_{2}+1$. Then from $(2.8),\left(2.3_{1}\right),\left(2.5_{1}\right)$, and $\left(2.7_{1}\right)$ we get

$$
\chi_{2}=1+\sum_{\lambda=\gamma_{5}+2}^{\infty} A\left(2^{\lambda}\right)=1+(-1)\left(\sum_{k=1}^{5} b_{k}-m\right) / 2 \cdot 2^{\gamma-4 \alpha-4} .
$$

(11) Let $\alpha \geq \gamma_{5}=\gamma_{4} \geq \gamma_{3} \geq \gamma_{2}+2$. Then from (2.8), (2.3. $)$, (2.4), $\left(2.5_{1}\right),(2.7)$, and $\left(2.7_{1}\right)$ we get

$$
\begin{gathered}
\chi_{2}=1+\sum_{\lambda=\gamma_{2}+2}^{\gamma_{3}} A\left(2^{\lambda}\right)+\sum_{\lambda=\gamma_{5}+2}^{\infty} A\left(2^{\lambda}\right)= \\
=1+\sum_{\lambda=\gamma_{2}+2}^{\gamma_{3}}(-1)^{\left(b_{1}+b_{2}\right) / 2} \cdot 2^{\gamma_{2}+1-\lambda}+(-1)^{\left(\sum_{k=1}^{5} b_{k}-m\right) / 2} \cdot 2^{\gamma-4 \alpha-4} .
\end{gathered}
$$

(12) Let $\alpha \geq \gamma_{5}>\gamma_{4} \geq \gamma_{3} \geq \gamma_{2}+2$. Then from (2.8), (2.3. $)$, (2.4), $\left(2.5_{1}\right),(2.6),(2.7)$, and $\left(2.7_{1}\right)$ we get

$$
\begin{gathered}
\chi_{2}=1+\sum_{\lambda=\gamma_{2}+2}^{\gamma_{3}} A\left(2^{\lambda}\right)+\sum_{\lambda=\gamma_{4}+2}^{\gamma_{5}} A\left(2^{\lambda}\right)+ \\
+\sum_{\lambda=\gamma_{5}+2}^{\infty} A\left(2^{\lambda}\right)=1+\sum_{\lambda=\gamma_{2}+2}^{\gamma_{3}}(-1)^{\left(b_{1}+b_{2}\right) / 2} \cdot 2^{\gamma_{2}+1-\lambda}+ \\
+\sum_{\lambda=\gamma_{4}+2}^{\gamma_{5}}(-1)\left(\sum_{k=1}^{4} b_{k}\right) / 2 \cdot 2^{\gamma-\gamma_{5}-3 \lambda+3}+(-1)^{\left(\sum_{k=1}^{5} b_{k}-m\right) / 2} \cdot 2^{\gamma-4 \alpha-4} .
\end{gathered}
$$

(13) Let $\alpha \geq \gamma_{5}>\gamma_{4} \geq \gamma_{3}=\gamma_{2}$ or $\alpha \geq \gamma_{5}>\gamma_{4} \geq \gamma_{3}=\gamma_{2}+1$. Then from (2.8), (2.3 $),\left(2.5_{1}\right),(2.6),(2.7)$, and (2.7 $)$ we get

$$
\begin{gathered}
\chi_{2}=1+\sum_{\lambda=\gamma_{4}+2}^{\gamma_{5}} A\left(2^{\lambda}\right)+\sum_{\lambda=\gamma_{5}+2}^{\infty} A\left(2^{\lambda}\right)= \\
=1+\sum_{\lambda=\gamma_{4}+2}^{\gamma_{5}}(-1)\left(\sum_{k=1}^{4} b_{k}\right) / 2 \cdot 2^{\sum_{k=2}^{4} \gamma_{k}-3 \lambda+3}+(-1)\left(\sum_{k=1}^{5} b_{k}-m\right) / 2 \cdot 2^{\gamma-4 \alpha-4} .
\end{gathered}
$$

Calculating the sums in the right-hand sides of the above given equalities, we complete the proof of the lemma.

Lemma 11. Let $p>2, p^{\beta}\left\|n, p^{\ell_{k}}\right\| a_{k}(k=1, \ldots, 5), \max \ell_{k}=\bar{\ell}, \min \ell_{k}=$ $\underline{\ell}=0, \bar{\ell} \geq \ell^{\prime \prime \prime} \geq \ell^{\prime \prime} \geq \ell^{\prime} \geq \underline{\ell}=0, \ell=\sum_{k=1}^{5} \ell_{k}=\bar{\ell}+\ell^{\prime \prime \prime}+\ell^{\prime \prime}+\ell^{\prime}+\underline{\ell}$, $\eta\left(\ell^{\prime}\right)=1$ if $2 \mid \ell^{\prime}$ and $\eta\left(\ell^{\prime}\right)=0$ if $2 \dagger \ell^{\prime}$. Then

$\chi_{p}=\left(1-p^{-1}\right)(\beta+1)$ for $\ell^{\prime} \geq \beta+1, p \equiv 1(\bmod 4)$, 
$=\left(1+p^{-1}\right)$ for $\ell^{\prime} \geq \beta+1, p \equiv 3(\bmod 4), 2 \mid \beta$,

$=0$ for $\ell^{\prime} \geq \beta+1, p \equiv 3(\bmod 4), 2 \dagger \beta$;

$=\left(1-p^{-1}\right) \ell^{\prime}+\left(1+p^{-1}\right)\left(1-p^{-\left(\beta-\ell^{\prime}+1\right)}\right)$

for $\ell^{\prime} \leq \beta<\ell^{\prime \prime}, p \equiv 1(\bmod 4)$,

$\left.=\left(1+p^{-1}\right) \eta\left(\ell^{\prime}\right)-(-1)^{\ell^{\prime}}(1+p) p^{-\left(\beta-\ell^{\prime}+2\right)}\right)$

for $\ell^{\prime} \leq \beta<\ell^{\prime \prime}, p \equiv 3(\bmod 4)$,

$=\left(1-p^{-1}\right) \ell^{\prime}+\left(1+p^{-1}\right)-p^{-\left(\ell^{\prime \prime}-\ell^{\prime}\right)}\left\{\left(1+p^{\left.-2\left(\beta-\ell^{\prime \prime}\right)-1\right)}\right)(p+1)^{-1}+\right.$

$\left.+p^{-2\left(\beta-\ell^{\prime \prime}\right)-3}\right\}$ for $\ell^{\prime \prime} \leq \beta<\ell^{\prime \prime \prime}, p \equiv 1(\bmod 4)$,

$=\left(1+p^{-1}\right) \eta\left(\ell^{\prime}\right)-(-1)^{\ell^{\prime}} p^{-\left(\ell^{\prime \prime}-\ell^{\prime}+1\right)}\left\{\left(1+(p-1)\left(p^{2}+1\right)^{-1} \times\right.\right.$

$\left.\times\left(1-(-1)^{\beta+\ell^{\prime \prime}} p^{-2\left(\beta-\ell^{\prime \prime}\right)}\right)-(-1)^{\beta+\ell^{\prime \prime}} p^{-2\left(\beta-\ell^{\prime \prime}+1\right)}\right\}$

for $\ell^{\prime \prime} \leq \beta<\ell^{\prime \prime \prime}, p \equiv 3(\bmod 4)$,

$=\left(1-p^{-1}\right) \ell^{\prime}+\left(1+p^{-1}\right)-p^{-\left(\ell^{\prime \prime}-\ell^{\prime}\right)}\left\{\left(1+p^{-2\left(\ell^{\prime \prime \prime}-\ell^{\prime \prime}\right)-1}\right)(p+1)^{-1}-\right.$

$-p^{-2\left(\ell^{\prime \prime \prime}-\ell^{\prime \prime}\right)-1}\left(1-p^{-3\left(\beta-\ell^{\prime \prime \prime}\right)}\left(p^{2}+p+1\right)^{-1}\right\}-p^{-3(\beta+1)+\ell-\bar{\ell}-1}$

for $\ell^{\prime \prime \prime} \leq \beta<\bar{\ell}, p \equiv 1(\bmod 4)$,

$=\left(1+p^{-1}\right) \eta\left(\ell^{\prime}\right)-(-1)^{\ell^{\prime}} p^{-\left(\ell^{\prime \prime}-\ell^{\prime}+1\right)}\left\{\left(1+(p-1)\left(p^{2}+1\right)^{-1} \times\right.\right.$

$\left.\times\left(1-(-1)^{\ell^{\prime \prime \prime}+\ell^{\prime \prime}} p^{-2\left(\beta-\ell^{\prime \prime}\right)}\right)\right\}+(-1)^{\ell-\bar{\ell}} p^{\ell-\bar{\ell}-3 \ell^{\prime \prime \prime}-1} \times$

$\times\left\{\left(1-p^{-3\left(\beta-\ell^{\prime \prime \prime}\right)}\right)\left(p^{2}+p+1\right)^{-1}-p^{-3\left(\beta-\ell^{\prime \prime \prime}+1\right)}\right\}$

for $\ell^{\prime \prime \prime} \leq \beta<\bar{\ell}, p \equiv 3(\bmod 4)$,

$=\left(1-p^{-1}\right) \ell^{\prime}+\left(1+p^{-1}\right)-p^{-\left(\ell^{\prime \prime}-\ell^{\prime}\right)}\left\{\left(1+p^{-2\left(\ell^{\prime \prime \prime}-\ell^{\prime \prime}\right)-1}\right)(p+1)^{-1}-\right.$

$-p^{-2\left(\ell^{\prime \prime \prime}-\ell^{\prime \prime}\right)-1}\left(1-p^{-3\left(\bar{\ell}-\ell^{\prime \prime \prime}\right)}\left(p^{2}+p+1\right)^{-1}\right\}+$

$+p^{\ell-4 \bar{\ell}-1}\left\{\left(1-p^{-4(\beta-\bar{\ell})}\right)\left(p^{3}+p^{2}+p+1\right)^{-1}-p^{-4(\beta-\bar{\ell}+1)}\right\}$

for $\beta \geq \bar{\ell}, p \equiv 1(\bmod 4)$,

$=\left(1+p^{-1}\right) \eta\left(\ell^{\prime}\right)-(-1)^{\ell^{\prime}} p^{-\left(\ell^{\prime \prime}-\ell^{\prime}+1\right)}\left\{\left(1+(p-1)\left(p^{2}+1\right)^{-1} \times\right.\right.$

$\left.\times\left(1-(-1)^{\ell^{\prime \prime \prime}+\ell^{\prime \prime}} p^{-2\left(\ell^{\prime \prime \prime}-\ell^{\prime \prime}\right)}\right)\right\}+(-1)^{\ell-\bar{\ell}} p^{\ell-\bar{\ell}-3 \ell^{\prime \prime \prime}-1} \times$

$\times\left\{\left(1-p^{-3\left(\bar{\ell}-\ell^{\prime \prime \prime}\right)}\right)\left(p^{2}+p+1\right)^{-1}-p^{-3\left(\bar{\ell}-\ell^{\prime \prime \prime}\right)}\left(1-(-1)^{\bar{\ell}+\beta} p^{-4(\beta-\bar{\ell})} \times\right.\right.$

$\left.\times(p-1)\left(p^{4}+1\right)^{-1}\right\}+(-1)^{\beta+\ell} p^{\ell-4 \beta-5}$ for $\beta \geq \bar{\ell}, p \equiv 3(\bmod 4)$. 
Proof. I. Let $2 \nmid q$ and $q=\left(q, a_{k}\right) q_{k}(k=1, \ldots, 5)$. Then from (1.14) and Lemmas 1 and 2 it follows that

$$
\begin{aligned}
A(q) & =q^{-10} \sum_{h \bmod q}^{\prime} e\left(-\frac{h n}{q}\right) \prod_{k=1}^{5}\left(q, a_{k}\right)^{2} S^{2}\left(\frac{a_{k}}{\left(q, a_{k}\right)} h, q_{k}\right)= \\
& =q^{-10} \sum_{h \bmod q}^{\prime} e\left(-\frac{h n}{q}\right) \prod_{k=1}^{5}\left(q, a_{k}\right)^{2}\left(\frac{-1}{q_{k}}\right) q_{k}= \\
& =q^{-5} \sum_{h \bmod q}^{\prime} e\left(-\frac{h n}{q}\right) \prod_{k=1}^{5}\left(q, a_{k}\right)\left(\frac{-1}{q_{k}}\right)
\end{aligned}
$$

where, putting $q=p^{\lambda}$ and taking into account that $\left(a_{1}, \ldots, a_{5}\right)=1$, we get

$$
\begin{aligned}
A\left(p^{\lambda}\right) & =p^{-5 \lambda} \sum_{h \bmod p^{\lambda}}^{\prime} e\left(-\frac{h n}{p^{\lambda}}\right) \prod_{k=1}^{5}\left(p^{\lambda}, a_{k}\right)\left(\frac{-1}{p^{\lambda} /\left(p^{\lambda}, a_{k}\right)}\right)= \\
& =\left(\frac{-1}{p}\right)^{\lambda+\min (\lambda, \bar{\ell})+\min \left(\lambda, \ell^{\prime \prime \prime}\right)+\min \left(\lambda, \ell^{\prime \prime}\right)+\min \left(\lambda, \ell^{\prime}\right)} \times \\
& \times p^{\min (\lambda, \bar{\ell})+\min \left(\lambda, \ell^{\prime \prime \prime}\right)+\min \left(\lambda, \ell^{\prime \prime}\right)+\min \left(\lambda, \ell^{\prime}\right)} p^{-5 \lambda} c\left(n, p^{\lambda}\right) .
\end{aligned}
$$

It follows from (2.9) and Lemma 4 that

(1) for $\lambda \leq \ell^{\prime}$

$$
\begin{aligned}
A\left(p^{\lambda}\right) & =\left(\frac{-1}{p}\right)^{\lambda}\left(1-p^{-1}\right) \text { if } \lambda<\beta+1, \\
& =-\left(\frac{-1}{p}\right)^{\beta+1} p^{-1} \text { if } \lambda=\beta+1
\end{aligned}
$$

(2) for $\ell^{\prime}<\lambda \leq \ell^{\prime \prime}$

$$
\begin{aligned}
A\left(p^{\lambda}\right) & =\left(\frac{-1}{p}\right)^{\ell^{\prime}} p^{-\left(\lambda-\ell^{\prime}\right)}\left(1-p^{-1}\right) \text { if } \lambda<\beta+1, \\
& =-\left(\frac{-1}{p}\right)^{\ell^{\prime}} p^{\ell^{\prime}-\beta-2} \text { if } \lambda=\beta+1 ;
\end{aligned}
$$

(3) for $\ell^{\prime \prime}<\lambda \leq \ell^{\prime \prime \prime}$

$$
\begin{aligned}
A\left(p^{\lambda}\right) & =\left(\frac{-1}{p}\right)^{\lambda+\ell^{\prime \prime}+\ell^{\prime}} p^{-2 \lambda+\ell^{\prime \prime} \ell^{\prime}}\left(1-p^{-1}\right) \text { if } \lambda<\beta+1, \\
& =-\left(\frac{-1}{p}\right)^{\beta+1+\ell^{\prime \prime}+\ell^{\prime}} p^{-2 \beta+\ell^{\prime \prime}+\ell^{\prime}-3} \text { if } \lambda=\beta+1 ;
\end{aligned}
$$


(4) for $\ell^{\prime \prime \prime}<\lambda \leq \bar{\ell}$

$$
\begin{aligned}
A\left(p^{\lambda}\right) & =\left(\frac{-1}{p}\right)^{\ell-\bar{\ell}} p^{\ell-\bar{\ell}-3 \lambda}\left(1-p^{-1}\right) \text { if } \lambda<\beta+1, \\
& =-\left(\frac{-1}{p}\right)^{\ell-\bar{\ell}} p^{\ell-\bar{\ell}} \text { if } \lambda=\beta+1 ;
\end{aligned}
$$

(5) for $\lambda>\bar{\ell}$

$$
\begin{aligned}
A\left(p^{\lambda}\right) & =\left(\frac{-1}{p}\right)^{\lambda+\ell} p^{\ell-4 \lambda}\left(1-p^{-1}\right) \text { if } \lambda<\beta+1, \\
& =-\left(\frac{-1}{p}\right)^{\beta+\ell+1} p^{\ell-4 \beta-5} \text { if } \lambda=\beta+1 .
\end{aligned}
$$

In all the above-mentioned cases

$$
A\left(p^{\lambda}\right)=0 \text { if } \lambda>\beta+1 .
$$

II. According to (1.15) we have

$$
\begin{aligned}
\chi_{p} & =1+\sum_{\lambda=1}^{\ell^{\prime}} A\left(p^{\lambda}\right)+\sum_{\lambda=\ell^{\prime}+1}^{\ell^{\prime \prime}} A\left(p^{\lambda}\right)+\sum_{\lambda=\ell^{\prime \prime}+1}^{\ell^{\prime \prime \prime}} A\left(p^{\lambda}\right)+ \\
& +\sum_{\lambda=\ell^{\prime \prime \prime}+1}^{\bar{\ell}} A\left(p^{\lambda}\right)+\sum_{\lambda=\bar{\ell}+1}^{\infty} A\left(p^{\lambda}\right) .
\end{aligned}
$$

Consider the following cases:

(1) Let $=\ell^{\prime} \geq \beta+1$. Then from (2.16), (2.10), $\left(2.10_{1}\right)$, and (2.15) we get

$$
\chi_{p}=1+\sum_{\lambda=1}^{\beta}\left(\frac{-1}{p}\right)^{\lambda}\left(1-p^{-1}\right)-\left(\frac{-1}{p}\right)^{\beta+1} p^{-1} .
$$

(2) Let $\ell^{\prime} \leq \beta<\ell^{\prime \prime}$. Then from (2.16), (2.10) (2.11), (2.11 $)$, and (2.15) we get

$$
\begin{gathered}
\chi_{p}=1+\sum_{\lambda=1}^{\ell^{\prime}}\left(\frac{-1}{p}\right)^{\lambda}\left(1-p^{-1}\right)+\sum_{\lambda=\ell^{\prime}+1}^{\beta}\left(\frac{-1}{p}\right)^{\ell^{\prime}} p^{-\left(\lambda-\ell^{\prime}\right)}\left(1-p^{-1}\right)- \\
-\left(\frac{-1}{p}\right)^{\ell^{\prime}} p^{-\left(\beta-\ell^{\prime}+1\right)} .
\end{gathered}
$$


(3) Let $\ell^{\prime \prime} \leq \beta<\ell^{\prime \prime \prime}$. Then from (2.16), (2.10), (2.11), (2.12), $\left(2.12_{1}\right)$, and (2.15) we get

$$
\begin{gathered}
\chi_{p}=1+\sum_{\lambda=1}^{\ell^{\prime}}\left(\frac{-1}{p}\right)^{\lambda}\left(1-p^{-1}\right)+\sum_{\lambda=\ell^{\prime}+1}^{\ell^{\prime \prime}}\left(\frac{-1}{p}\right)^{\ell^{\prime}} p^{-\left(\lambda-\ell^{\prime}\right)}\left(1-p^{-1}\right)+ \\
+\sum_{\lambda=\ell^{\prime \prime}+1}^{\beta}\left(\frac{-1}{p}\right)^{\lambda+\ell^{\prime \prime}+\ell^{\prime}} p^{-2 \lambda+\ell^{\prime \prime}+\ell^{\prime}}\left(1-p^{-1}\right)-\left(\frac{-1}{p}\right)^{\beta+1+\ell^{\prime \prime}+\ell^{\prime}} p^{-2 \beta+\ell^{\prime \prime}+\ell^{\prime}+3} .
\end{gathered}
$$

(4) Let $\ell^{\prime \prime \prime} \leq \beta<\bar{\ell}$. Then from (2.16), (2.10), (2.11), (2.12), (2.13), $\left(2.13_{1}\right)$, and (2.15) we get

$$
\begin{aligned}
\chi_{p}= & +\sum_{\lambda=1}^{\ell^{\prime}}\left(\frac{-1}{p}\right)^{\lambda}\left(1-p^{-1}\right)+\sum_{\lambda=\ell^{\prime}+1}^{\ell^{\prime \prime}}\left(\frac{-1}{p}\right)^{\ell^{\prime}} p^{-\left(\lambda-\ell^{\prime}\right)}\left(1-p^{-1}\right)+ \\
& +\sum_{\lambda=\ell^{\prime \prime}+1}\left(\frac{-1}{p}\right)^{\lambda+\ell^{\prime \prime}+\ell^{\prime}} p^{-2 \lambda+\ell^{\prime \prime}+\ell^{\prime}}\left(1-p^{-1}\right)+ \\
+ & \sum_{\lambda=\ell^{\prime \prime \prime}+1}^{\beta}\left(\frac{-1}{p}\right)^{\ell-\bar{\ell}} p^{\ell-\bar{\ell}-3 \lambda}\left(1-p^{-1}\right)-\left(\frac{-1}{p}\right)^{\ell-\bar{\ell}} p^{\ell-\bar{\ell}-3 \beta-4} .
\end{aligned}
$$

(5) Let $\beta \geq \bar{\ell}$. Then from (2.16), (2.10), (2.11), (2.12), (2.13), (2.14), $\left(2.14_{1}\right)$, and $(2.15)$ we get

$$
\begin{gathered}
\chi_{p}=1+\sum_{\lambda=1}^{\ell^{\prime}}\left(\frac{-1}{p}\right)^{\lambda}\left(1-p^{-1}\right)+\sum_{\lambda=\ell^{\prime}+1}^{\ell^{\prime \prime}}\left(\frac{-1}{p}\right)^{\ell^{\prime}}\left(1-p^{-1}\right) p^{-\left(\lambda-\ell^{\prime}\right)}+ \\
+\sum_{\lambda=\ell^{\prime \prime}+1}^{\ell^{\prime \prime \prime}}\left(\frac{-1}{p}\right)^{\lambda+\ell^{\prime \prime}+\ell^{\prime}} p^{\ell^{\prime \prime}+\ell^{\prime}-2 \lambda}\left(1-p^{-1}\right)+\sum_{\lambda=\ell^{\prime \prime \prime}+1}^{\bar{\ell}}\left(\frac{-1}{p}\right)^{\ell-\bar{\ell}} p^{\ell-\bar{\ell}-3 \lambda}\left(1-p^{-1}\right)+ \\
+\sum_{\lambda=\bar{\ell}+1}^{\beta}\left(\frac{-1}{p}\right)^{\lambda+\ell} p^{\ell-4 \lambda}\left(1-p^{-1}\right)-\left(\frac{-1}{p}\right)^{\beta+\ell+1} p^{\ell-4 \beta-5} .
\end{gathered}
$$

Calculating the sums in the right-hand sides of the above-mentioned equalities, we complete the proof of the lemma.

Theorem 1. Let $n=2^{\alpha} m=2^{\alpha} u v, u=\prod_{\substack{p \mid n \\ p \nmid 2 \Delta}} p^{\beta}, v=\prod_{\substack{p|n \\ p| \Delta, p>2}} p^{\beta}$. Then

$$
\rho(n ; f)=\frac{2^{4 \alpha+6} v^{4}}{5 \Delta^{1 / 2}} \chi_{2} \prod_{\substack{p \mid \Delta \\ p>2}} \chi_{p} \prod_{p \mid \Delta, p>2}\left(1-\left(\frac{-1}{p}\right) p^{-5}\right)^{-1} \sum_{d_{1} d_{2}=u}\left(\frac{-1}{d_{1}}\right) d_{2}^{4},
$$


where the values of $\chi_{2}$ and $\chi_{p}$ are given in Lemmas 10 and 11.

Proof. Let $p>2 p^{\beta} \| n, p \nmid \Delta$ (i.e., $\ell=0$ ). In (2.17) putting $\ell=0$, we get

$$
\begin{gathered}
\chi_{p}=1+\sum_{\lambda=1}^{\beta}\left(\frac{-1}{p}\right)^{\lambda} p^{-4 \lambda}\left(1-p^{-1}\right)-\left(\frac{-1}{p}\right)^{\beta+1} p^{-4 \beta-5}= \\
=1+\sum_{\lambda=1}^{\beta}\left(\frac{-1}{p}\right)^{\lambda} p^{-4 \lambda}-\left(\frac{-1}{p}\right) p^{-5} \sum_{\lambda=1}^{\beta}\left(\frac{-1}{p}\right)^{\lambda-1} p^{-4(\lambda-1)}- \\
-\left(\frac{-1}{p}\right)^{\beta+1} p^{-4 \beta-5}=\sum_{\lambda=0}^{\beta}\left(\frac{-1}{p}\right)^{\lambda} p^{-4 \lambda}-\left(\frac{-1}{p}\right) p^{-5} \sum_{\lambda=0}^{\beta}\left(\frac{-1}{p}\right)^{\lambda} p^{-4 \lambda}= \\
=\left(1-\left(\frac{-1}{p}\right) p^{-5}\right) \sum_{\lambda=0}^{\beta}\left(\frac{-1}{p}\right)^{\lambda} p^{-4 \lambda}= \\
=\left(1-\left(\frac{-1}{p}\right) p^{-5}\right) \sum_{d \mid p^{\beta}}\left(\frac{-1}{d}\right) p^{-4} .
\end{gathered}
$$

For $p \nmid \Delta n$, i.e., for $\beta=0$, from (2.18) we get

$$
\chi_{p}=1-\left(\frac{-1}{p}\right) p^{-5} \text {. }
$$

Thus it follows from Lemma 5, (2.18), and (2.19) that

$$
\begin{gathered}
\sum_{q=1}^{\infty} A(q)=\chi_{2} \prod_{\substack{p \mid \Delta \\
p>2}} \chi_{p} \prod_{\substack{p \mid n \\
p \nmid 2 \Delta}} \chi_{p} \prod_{\substack{p \dagger \Delta n \\
p>2}} \chi_{p}= \\
=\chi_{2} \prod_{\substack{p \mid \Delta \\
p>2}} \chi_{p} \prod_{\substack{p \mid n \\
p \nmid 2 \Delta}}\left\{\left(1-\left(\frac{-1}{p}\right) p^{-5}\right) \sum_{d \mid p^{\beta}}\left(\frac{-1}{d}\right) d^{-4}\right\} \prod_{\substack{p \dagger \Delta n \\
p>2}}\left(1-\left(\frac{-1}{p}\right) p^{-5}\right)= \\
=\chi_{2} \prod_{\substack{p \mid \Delta \\
p>2}} \chi_{p} \prod_{p>2}\left(1-\left(\frac{-1}{p}\right) p^{-5}\right) \prod_{\substack{p \mid \Delta \\
p>2}}\left(1-\left(\frac{-1}{p}\right) p^{-5}\right)^{-1} \sum_{d \mid u}\left(\frac{-1}{d}\right) d^{4}= \\
=\chi_{2} \prod_{\substack{p \mid \Delta \\
p>2}} \chi_{p} \mathcal{L}^{-1}(5,-1) \prod_{\substack{p \mid \Delta \\
p>2}}\left(1-\left(\frac{-1}{p}\right) p^{-5}\right)^{-1} \frac{1}{u^{4}} \sum_{d_{1} d_{2}=u}\left(\frac{-1}{d_{1}}\right) d_{2}^{4}, \quad(2.20)
\end{gathered}
$$

where $\mathcal{L}(5,-1)$ is the Dirichlet $\mathcal{L}$-function and it is well known that

$$
\mathcal{L}(5,-1)=\frac{5 \pi^{4}}{2^{6} \cdot 4 !} .
$$

Thus the lemma follows from (1.13), (2.20), and (2.21). 


\section{Formulas FOR $r(n ; f)$}

As an example let us consider the quadratic form

$$
f=x_{1}^{2}+\cdots+x_{8}^{2}+4\left(x_{9}^{2}+x_{10}^{2}\right)
$$

in which

$$
\begin{gathered}
a_{1}=\cdots a_{4}=1, \quad a_{5}=4, \quad b_{1}=\cdots=b_{5}=1, \\
\gamma_{5}=2, \quad \gamma_{4}=\gamma_{3}=\gamma_{2}=\gamma_{1}=0, \quad \gamma=2, \quad a=4, \quad \Delta=2^{4} .
\end{gathered}
$$

Lemma 12. The function

$$
\begin{gathered}
\psi(\tau ; f)=\vartheta_{00}^{8}(\tau ; 0,2) \vartheta_{00}^{2}(\tau ; 0,8)-\theta(\tau ; f)+\frac{42}{5 \cdot 512 \pi^{4}} \Psi_{2}(\tau ; 8,8 ; 0,0 ; 0,0 ; 4,4)- \\
-\frac{544}{5 \cdot 8192 \pi^{4}} \Psi_{2}(\tau ; 0,0 ; 0,0 ; 0,0 ; 4,4)+\frac{3}{5 \cdot 128 \pi^{4}} \Psi_{2}(\tau ; 4,4 ; 0,0 ; 0,0 ; 2,2)+ \\
\quad+\frac{2}{32 \pi^{3} i} \Psi_{3}(\tau ; 4,0,4,0 ; 0,1,1,1 ; 0,0,0,0 ; 2,4,2,4)+ \\
\quad+\frac{24}{512 \pi^{3} i} \Psi_{4}(\tau ; 8,8,8,0 ; 1,1,1,1 ; 0,0,0,0 ; 4,4,4,4)
\end{gathered}
$$

is an entire modular form of weight 5 and character $\chi(\delta)=\operatorname{sgn} \delta\left(\frac{-1}{|\delta|}\right)$ for $\Gamma_{0}(16)$.

Proof. According to Lemmas 7 and 8, the first two summands in (3.1) are entire modular forms of weight 5 and character $\chi(\delta)=\operatorname{sgn} \delta\left(\frac{-1}{|\delta|}\right)$ for $\Gamma_{0}(16)$.

Put $N=4$. Then it is obvious that the following five summands in (3.1) satisfy the condition (a) from Lemma 9.

If $\alpha \delta \equiv 1(\bmod 16)$, then $\alpha \delta \equiv 1(\bmod 4)$, i.e.,

$$
\alpha \equiv \pm 1(\bmod 4) \text { and respectively } \delta \equiv \pm 1(\bmod 4) .
$$

In the third, fourth, and fifth summands from (3.1) we have

$$
\left(\frac{N_{1} N_{2}}{|\delta|}\right)=1 \text { and }\left(\frac{\Delta}{|\delta|}\right)=1 .
$$

By (1.16), (1.6), (1.7), and (1.10) we have

$$
\begin{gathered}
\Psi_{2}(\tau ; 8 \alpha, 8 \alpha ; 0,0 ; 0,0 ; 4,4)=\frac{1}{8} \vartheta_{8 \alpha, 0}^{(4)}(\tau ; 0,8) \vartheta_{8 \alpha, 0}(\tau ; 0,8)-\frac{3}{8} \vartheta_{8 \alpha, 0}^{\prime \prime 2}(\tau ; 0,8)= \\
=\frac{1}{8} \vartheta_{ \pm 8+8(\alpha \mp 1), 0}^{(4)}(\tau ; 0,8) \vartheta_{ \pm 8+8(\alpha \mp 1), 0}(\tau ; 0,8)-\frac{3}{8} \vartheta_{ \pm 8+8(\alpha \mp 1), 0}^{\prime \prime 2}(\tau ; 0,8)= \\
=\frac{1}{8} \vartheta_{ \pm 8,0}^{(4)}(\tau ; 4(\alpha \mp 1), 8) \vartheta_{ \pm 8,0}(\tau ; 4(\alpha \mp 1), 8)-\frac{3}{8} \vartheta_{ \pm 8,0}^{\prime \prime 2}(\tau ; 4(\alpha \mp 1), 8)= \\
=\frac{1}{8} \vartheta_{ \pm 8,0}^{(4)}(\tau ; 0,8) \vartheta_{ \pm 8,0}(\tau ; 0,8)-\frac{3}{8} \vartheta_{ \pm 8,0}^{\prime \prime 2}(\tau ; 0,8)=\frac{1}{8} \vartheta_{80}^{(4)}(\tau ; 0,8) \times
\end{gathered}
$$




$$
\times \vartheta_{80}(\tau ; 0,8)-\frac{3}{8} \vartheta_{80}^{\prime \prime 2}(\tau ; 0,8)=\Psi_{2}(\tau ; 8,8 ; 0,0 ; 0,0 ; 4,4)
$$

and similarly

$$
\begin{gathered}
\Psi_{2}(\tau ; 4 \alpha, 4 \alpha ; 0,0 ; 0,0 ; 2,2)=\frac{1}{2} \vartheta_{4 \alpha, 0}^{(4)}(\tau ; 0,4) \vartheta_{4 \alpha, 0}(\tau ; 0,4)-\frac{3}{2} \vartheta_{4 \alpha, 0}^{\prime \prime 2}(\tau ; 0,4)= \\
=\frac{1}{2} \vartheta_{40}^{(4)}(\tau ; 0,4) \vartheta_{40}(\tau ; 0,4)-\frac{3}{2} \vartheta_{40}^{\prime \prime 2}(\tau ; 0,4)= \\
=\Psi_{2}(\tau ; 4,4 ; 0,0 ; 0,0 ; 2,2) .
\end{gathered}
$$

Hence, according to $(3.2)-(3.5)$ the functions $\Psi_{2}(\tau ; 8,8 ; 0,0 ; 0,0 ; 4,4)$ and $\Psi_{2}(\tau ; 4,4 ; 0,0 ; 0,0 ; 2,2)$ satisfy also the condition (b) from Lemma 9 . Thus they are entire modular forms of weight 5 and character $\chi(\delta)=\operatorname{sgn} \delta\left(\frac{-1}{|\delta|}\right)$ for $\Gamma_{0}(16)$.

Analogously, by (1.17), (1.18), (1.6), (1.7), and (1.10) we obtain

$$
\begin{gathered}
\Psi_{3}(\tau ; 4 \alpha, 0,4 \alpha, 0 ; 0,1,1,1 ; 0,0,0,0 ; 2,4,2,4)=\left\{\frac{1}{2} \vartheta_{4 \alpha, 0}^{\prime \prime}(\tau ; 0,4) \vartheta_{01}(\tau ; 0,8)-\right. \\
\left.-\frac{1}{4} \vartheta_{4 \alpha, 0}(\tau ; 0,4) \vartheta_{01}^{\prime \prime}(\tau ; 0,8)\right\} \vartheta_{4 \alpha, 1}^{\prime \prime}(\tau ; 0,4) \vartheta_{01}(\tau ; 0,8)= \\
= \pm\left\{\frac{1}{2} \vartheta_{40}^{\prime \prime}(\tau ; 0,4) \vartheta_{01}(\tau ; 0,8)-\frac{1}{4} \vartheta_{40}(\tau ; 0,4) \vartheta_{01}^{\prime \prime}(\tau ; 0,8)\right\} \vartheta_{41}^{\prime}(\tau ; 0,4) \times \\
\times \vartheta_{01}(\tau ; 0,8)= \pm \Psi_{3}(\tau ; 4,0,4,0 ; 0,1,1,1 ; 0,0,0,0 ; 2,4,2,4), \\
\Psi_{4}(\tau ; 8 \alpha, 8 \alpha, 8 \alpha, 0 ; 1,1,1,1 ; 0,0,0,0 ; 4,4,4,4)= \\
=\vartheta_{8 \alpha, 1}^{\prime 3}(\tau ; 0,8) \vartheta_{01}(\tau ; 0,8)= \pm \vartheta_{81}^{\prime 3}(\tau ; 0,8) \vartheta_{01}(\tau ; 0,8)= \\
= \pm \Psi_{4}(\tau ; 8,8,8,0 ; 1,1,1,1 ; 0,0,0,0 ; 4,4,4,4) .
\end{gathered}
$$

In ((3.6) and (3.7) we have "+" if $\alpha \equiv 1(\bmod 4)$ and "-" if $\alpha \equiv-1$ $(\bmod 4)$. Hence the functions $\Psi_{3}(\tau ; 4,0,4,0 ; 0,1,1,1 ; 0,0,0,0 ; 2,4,2,4)$ and $\Psi_{4}(\tau ; 8,8,8,0 ; 1,1,1,1 ; 0,0,0,0 ; 4,4,4,4)$ also satisfy the condition (b) from Lemma 9 , since

$$
\left(\frac{\prod_{k=1}^{4} N_{k}}{|\delta|}\right)=1 \text { and } \operatorname{sgn} \delta\left(\frac{-1}{|\delta|}\right)= \begin{cases}1 & \text { if } \delta \equiv 1(\bmod 4) \\ -1 & \text { if } \delta \equiv-1(\bmod 4)\end{cases}
$$

Thus, they are entire modular forms of weight 5 and character $\chi(\delta)=$ $\operatorname{sgn} \delta\left(\frac{-1}{|\delta|}\right)$ for $\Gamma_{0}(16)$.

\section{Theorem 2.}

$$
\begin{gathered}
\vartheta_{00}^{8}(\tau ; 0,2) \vartheta_{00}^{2}(\tau ; 0,8)=\theta(\tau ; f)-\frac{42}{5 \cdot 512 \pi^{4}} \Psi_{2}(\tau ; 8,8 ; 0,0 ; 0,0 ; 4,4)+ \\
+\frac{544}{5 \cdot 8192 \pi^{4}} \Psi_{2}(\tau ; 0,0 ; 0,0 ; 0,0 ; 4,4)-\frac{3}{5 \cdot 128 \pi^{4}} \Psi_{2}(\tau ; 4,4 ; 0,0 ; 0,0 ; 2,2)-
\end{gathered}
$$




$$
\begin{aligned}
& -\frac{2}{32 \pi^{3} i} \Psi_{3}(\tau ; 4,0,4,0 ; 0,1,1,1 ; 0,0,0,0 ; 2,4,2,4)- \\
& -\frac{24}{512 \pi^{3} i} \Psi_{4}(\tau ; 8,8,8,0 ; 1,1,1,1 ; 0,0,0,0 ; 4,4,4,4) .
\end{aligned}
$$

Proof. According to Lemma 6 , the function $\psi(r ; f)$ will be identically zero if all coefficients by $Q^{n}(n \leq 10)$ in its expansion by powers of $Q$ are zero.

I. In Theorem 1 and Lemma 10 put $n=2^{\alpha} u, m=u, v=1, \Delta=2^{4}$. Then

$$
\begin{aligned}
\rho(n ; f) & =\frac{16}{5} \sum_{d_{1} d_{2}=u}\left(\frac{-1}{d_{1}}\right) d_{2}^{4} \text { for } \alpha=0, \\
& =\frac{224}{5} \sum_{d_{1} d_{2}=u}\left(\frac{-1}{d_{1}}\right) d_{2}^{4} \text { for } \alpha=1 \\
& =\frac{4}{5}\left(2^{4 \alpha-1} \cdot 9+\left(\frac{-1}{u}\right)\right) \sum_{d_{1} d_{2}=u}\left(\frac{-1}{d_{1}}\right) d_{2}^{4} \text { for } \alpha \geq 2 .
\end{aligned}
$$

Calculating the values of $\rho(n ; f)$ by the above formulas for all $n \leq 10$, we get

$$
\begin{aligned}
\theta(\tau ; f) & =1+\frac{16}{5} Q+\frac{224}{5} Q^{2}+256 Q^{3}+\frac{4612}{5} Q^{4}+\frac{10016}{5} Q^{5}+3584 Q^{6}+ \\
& +7680 Q^{7}+\frac{73732}{5} Q^{8}+\frac{103696}{5} Q^{9}+\frac{140224}{5} Q^{10}+\cdots
\end{aligned}
$$

It follows from (1.8) that

$$
\begin{array}{r}
\vartheta_{00}^{8}(\tau ; 0,2) \vartheta_{00}^{2}(\tau ; 0,8)=1+16 Q+112 Q^{2}+448 Q^{3}+1140 Q^{4}+2080 Q^{5}+ \\
+3584 Q^{6}+7296 Q^{7}+13876 Q^{8}+20240 Q^{9}+27104 Q^{10}+\cdots .(3.10)
\end{array}
$$

II. From (1.8) and (1.9) we have

(1) $\frac{1}{8} \vartheta_{80}^{(4)}(\tau ; 0,8) \vartheta_{80}(\tau ; 0,8)=\frac{1}{8} 4096 \pi^{4} \sum_{m_{1}=-\infty}^{\infty}\left(2 m_{1}+1\right)^{4} Q^{\left(2 m_{1}+1\right)^{2}} \times$

$$
\begin{gathered}
\times \sum_{m_{2}=-\infty}^{\infty} Q^{\left(2 m_{1}+1\right)^{2}}=512 \pi^{4} \cdot 4 Q^{2}\left(1+81 Q^{8}+625 Q^{24}+\cdots\right) \times \\
\times\left(1+Q^{8}+Q^{24}+\cdots\right)=512 \pi^{4}\left(4 Q^{2}+328 Q^{10}+\cdots\right) \\
\frac{3}{8} \vartheta_{80}^{\prime \prime 2}(\tau ; 0,8)=\frac{3}{8} 4096 \pi^{4} \sum_{m_{1}=-\infty}^{\infty}\left(2 m_{1}+1\right)^{4} Q^{\left(2 m_{1}+1\right)^{2}} \times
\end{gathered}
$$




$$
\begin{aligned}
& \times \sum_{m_{2}=-\infty}^{\infty}\left(2 m_{2}+1\right)^{4} Q^{\left(2 m_{1}+1\right)^{2}}=3 \cdot 512 \pi^{4} \cdot 4 Q^{2} \times \\
& \times\left(1+9 Q^{8}+25 Q^{24}+\cdots\right)^{2}=512 \pi^{4}\left(12 Q^{2}+216 Q^{10}+\cdots\right) ; \\
& \text { (2) } \frac{1}{8} \vartheta_{00}^{(4)}(\tau ; 0,8) \vartheta_{00}(\tau ; 0,8)=\frac{1}{8} \cdot 2^{16} \pi^{4} \sum_{m_{1}=-\infty}^{\infty} m_{1}^{4} Q^{4 m_{1}^{2}} \sum_{m_{2}=-\infty}^{\infty} Q^{4 m_{2}^{2}}= \\
& =8192 \pi^{4}\left(2 Q^{4}+4 Q^{8}+32 Q^{16}+\cdots\right) \text {, } \\
& \frac{3}{8} \vartheta_{\infty}^{\prime \prime 2}(\tau ; 0,8)=\frac{3}{8} \cdot 2^{16} \pi^{4} \sum_{m_{1}=-\infty}^{\infty} m_{1}^{2} Q^{4 m_{1}^{2}} \sum_{m_{2}=-\infty}^{\infty} m_{2}^{2} Q^{4 m_{2}^{2}}= \\
& =8192 \pi^{4}\left(12 Q^{8}+96 Q^{20}+\cdots\right) \text {; } \\
& \text { (3) } \frac{1}{2} \vartheta_{40}^{(4)}(\tau ; 0,4) \vartheta_{40}(\tau ; 0,4)=\frac{1}{2} \cdot 256 \pi^{4} \sum_{m_{1}=-\infty}^{\infty}\left(2 m_{1}+1\right)^{4} Q^{\left(2 m_{1}+1\right)^{2} / 2} \times \\
& \times \sum_{m_{2}=-\infty}^{\infty} Q^{\left(2 m_{2}+1\right)^{2} / 2}= \\
& =128 \pi^{4}\left(4 Q+328 Q^{5}+324 Q^{9}+2504 Q^{13}+\cdots\right), \\
& \frac{3}{2} \vartheta_{40}^{\prime \prime 2}(\tau ; 0,4)=\frac{3}{2} \cdot 256 \pi^{4} \sum_{m_{1}=-\infty}^{\infty}\left(2 m_{1}+1\right)^{2} Q^{\left(2 m_{1}+1\right)^{2} / 2} \times \\
& \times \sum_{m_{2}=-\infty}^{\infty}\left(2 m_{2}+1\right)^{2} Q^{\left(2 m_{2}+1\right)^{2} / 2}= \\
& =128 \pi^{4}\left(12 Q+216 Q^{5}+972 Q^{9}+600 Q^{13}+\cdots\right) ; \\
& \text { (4) } \frac{1}{2} \vartheta_{40}^{\prime \prime}(\tau ; 0,4) \vartheta_{01}(\tau ; 0,8)=\frac{1}{2} \cdot 16 \pi^{2} \sum_{m_{1}=-\infty}^{\infty}\left(2 m_{1}+1\right)^{2} Q^{\left(2 m_{1}+1\right)^{2} / 2} \times \\
& \times \sum_{m_{3}=-\infty}^{\infty}(-1)^{m_{3}} Q^{4 m_{3}^{2}}=-8 \pi^{2} \cdot 2 Q^{1 / 2}\left(1+9 Q^{4}+25 Q^{12}+\cdots\right)\left(1-2 Q^{4}+\right. \\
& \left.+2 Q^{16}-\cdots\right)=-8 \pi^{2} \cdot 2 Q^{1 / 2}\left(1+7 Q^{4}-18 Q^{8}+25 Q^{12}+\cdots\right), \\
& \frac{1}{4} \vartheta_{40}(\tau ; 0,4) \vartheta_{01}^{\prime \prime}(\tau ; 0,8)=-\frac{1}{4} \sum_{m_{1}=-\infty}^{\infty} Q^{\left(2 m_{1}+1\right)^{2} / 2} \cdot 256 \pi^{2} \times \\
& \times \sum_{m_{3}=-\infty}^{\infty}(-1)^{m_{3}} m_{3}^{2} Q^{4 m_{3}^{2}}=-64 \pi^{2} \cdot 2 Q^{1 / 2}\left(1+Q^{4}+Q^{12}+\cdots\right)\left(-2 Q^{4}+\right. \\
& \left.+8 Q^{16}-\cdots\right)=-64 \pi^{2} \cdot 2 Q^{1 / 2}\left(-2 Q^{4}-2 Q^{8}+6 Q^{16}+\cdots\right),
\end{aligned}
$$




$$
\begin{gathered}
\vartheta_{41}^{\prime}(\tau ; 0,4) \vartheta_{01}(\tau ; 0,8)=-4 \pi i \sum_{m_{2}=-\infty}^{\infty}(-1)^{m_{2}} Q^{\left(2 m_{2}+1\right)^{2} / 2} \times \\
\times \sum_{m_{4}=-\infty}^{\infty}(-1)^{m_{4}} Q^{4 m_{4}^{2}}=4 \pi i \cdot 2 Q^{1 / 2}\left(1-3 Q^{4}+5 Q^{12}-\cdots\right)\left(1-2 Q^{4}+\right. \\
\left.+2 Q^{16}-\cdots\right)=4 \pi i \cdot 2 Q^{1 / 2}\left(1-5 Q^{4}+6 Q^{8}+5 Q^{12}-\cdots\right), \quad(3.19) \\
(5) \vartheta_{81}^{\prime 3}(\tau ; 0,8) \vartheta_{01}(\tau ; 0,8)=-512 \pi^{3} i \sum_{m_{1}, m_{2}, m_{3}=-\infty}^{\infty}(-1)^{m_{1}+m_{2}+m_{3}}\left(2 m_{1}+1\right) \times \\
\times\left(2 m_{2}+1\right)\left(2 m_{3}+1\right) Q^{\left(2 m_{1}+1\right)^{2}+\left(2 m_{2}+1\right)^{2}+\left(2 m_{3}+1\right)^{2}} \sum_{m_{4}=-\infty}^{\infty}(-1)^{m_{4}} Q^{4 m_{4}^{2}}= \\
=-512 \pi^{3} i\left(8 Q^{3}-16 Q^{7}-72 Q^{11}+\cdots\right) .
\end{gathered}
$$

III. From (3.4), (3.11), and (3.12) it follows that

$$
\frac{1}{512 \pi^{4}} \Psi_{2}(\tau ; 8,8 ; 0,0 ; 0,0 ; 4,4)=-8 Q^{2}+112 Q^{10}+\cdots .
$$

From (1.16), (3.13), and (3.14) it follows that

$$
\frac{1}{8192 \pi^{4}} \Psi_{2}(\tau ; 0,0 ; 0,0 ; 0,0 ; 4,4)=2 Q^{4}-8 Q^{8}+31 Q^{16}+\cdots .
$$

From (3.5), (3.15), and (3.16) it follows that

$$
\frac{1}{128 \pi^{4}} \Psi_{2}(\tau ; 4,4 ; 0,0 ; 0,0 ; 2,2)=-8 Q+112 Q^{5}+1904 Q^{13}-\cdots .
$$

From (3.6), (3.17)-(3.19) it follows that

$$
\begin{gathered}
-\frac{1}{32 \pi^{3} i} \Psi_{3}(\tau ; 4,0,4,0 ; 0,1,1,1 ; 0,0,0,0 ; 2,4,2,4)= \\
=4 Q+72 Q^{5}-444 Q^{9}+712 Q^{13}+\cdots .
\end{gathered}
$$

From (3.7) and (3.20) follows

$$
\begin{gathered}
-\frac{1}{512 \pi^{3} i} \Psi_{4}(\tau ; 8,8,8,0 ; 1,1,1,1 ; 0,0,0,0 ; 4,4,4,4)= \\
=8 Q^{3}-16 Q^{7}-72 Q^{11}+\cdots .
\end{gathered}
$$

According to (3.1), (3.9), (3.10), and (3.21)-(3.25) it is easy to verify that all coefficients by $Q^{n}(n \leq 10)$ in the expansion of the function $\psi(\tau ; f)$ by powers of $Q$ are zero. 
Theorem 2a. Let $n=2^{\alpha} u$. Then

$$
\begin{aligned}
& r(n ; f)=\frac{16}{5} \sum_{d_{1} d_{2}=u}\left(\frac{-1}{d}\right) d_{2}^{4}-\frac{12}{5} \sum_{\substack{x^{2}+y^{2}=2 u \\
2 \dagger x, 2 \dagger y, x>0 y>0}}\left(x^{4}-3 x^{2} y^{2}\right)+ \\
& \left.+8 \sum_{\substack{x^{2}+y^{2}+8\left(z^{2}+t^{2}\right)=2 u \\
2 \dagger x, 2 \dagger y, x>0, y>0}}(-1)^{(y-1) / 2+z+t}\left(x^{2}-8 z^{2}\right) y\right) \\
& \text { for } \alpha=0, u \equiv 1(\bmod 4), \\
& =\frac{16}{5} \sum_{d_{1} d_{2}=u}\left(\frac{-1}{d}\right) d_{2}^{4}+192 \sum_{\substack{x^{2}+y^{2}+z^{2}+4 t^{2}=u \\
2 \dagger x, 2 \dagger y, 2 \uparrow t, x>0, y>0, z>0}}(-1)^{(x+y+z-3) / 1+t} x y z \\
& \text { for } \alpha=0, u \equiv 3(\bmod 4), \\
& =\frac{224}{5} \sum_{d_{1} d_{2}=u}\left(\frac{-1}{d}\right) d_{2}^{4}-\frac{168}{5} \sum_{\substack{x^{2}+y^{2}=2 u \\
2 \dagger x, 2 \dagger y, x>0, y>0}}\left(x^{4}-3 x^{2} y^{2}\right) \\
& \text { for } \alpha=0=0, u \equiv 1(\bmod 4) \text {, } \\
& =\frac{224}{5} \sum_{d_{1} d_{2}=u}\left(\frac{-1}{d}\right) d_{2}^{4} \text { for } \alpha=1, u \equiv 3(\bmod 4), \\
& =\frac{4612}{5} \sum_{d_{1} d_{2}=u}\left(\frac{-1}{d}\right) d_{2}^{4}+\frac{1088}{5} \sum_{\substack{x^{2}+y^{2}=u \\
2 \dagger x, x>0, y=0}}\left(x^{4}-3 x^{2} y^{2}\right)+ \\
& +\frac{2176}{5} \sum_{\begin{array}{c}
x^{2}+y^{2}=u \\
x \neq y y(\bmod 2) \\
x>0, y>0
\end{array}}\left(x^{4}-3 x^{2} y^{2}\right) \text { for } \alpha=2, u \equiv 1(\bmod 8), \\
& =\frac{4612}{5} \sum_{d_{1} d_{2}=u}\left(\frac{-1}{d}\right) d_{2}^{4}+\frac{2176}{5} \sum_{\substack{x^{2}+y^{2}=u \\
x \neq y(\bmod 2) \\
x>0, y>0}}\left(x^{4}-3 x^{2} y^{2}\right) \\
& \text { for } \alpha=2, u \equiv 5(\bmod 8), \\
& =\frac{4}{5}\left(2^{4 \alpha-1} \cdot 9-1\right) \sum_{d_{1} d_{2}=u}\left(\frac{-1}{d}\right) d_{2}^{4} \text { for } \alpha \geq 2, u \equiv 3(\bmod 4) \text {, } \\
& =\frac{73732}{5} \sum_{d_{1} d_{2}=u}\left(\frac{-1}{d}\right) d_{2}^{4}+\frac{2176}{5} \sum_{\begin{array}{c}
x^{2}+y^{2}=2 u \\
2 \dagger x, 2 \dagger y, x>0, y>0
\end{array}}\left(x^{4}-3 x^{2} y^{2}\right)
\end{aligned}
$$




$$
\begin{gathered}
=\frac{4}{5}\left(2^{4 \alpha-1} \cdot 9+1\right) \sum_{d_{1} d_{2}=u}\left(\frac{-1}{d}\right) d_{2}^{4}+\frac{2176}{5} \sum_{\substack{x^{2}+y^{2}=2^{\alpha-2} u \\
2|x, 2| y, x>0, y>0}}\left(x^{4}-3 x^{2} y^{2}\right) \\
\text { for } 2 \nmid \alpha, \alpha>3, u \equiv 1(\bmod 4), \\
=\frac{4}{5}\left(2^{4 \alpha-1} \cdot 9+1\right) \sum_{d_{1} d_{2}=u}\left(\frac{-1}{d}\right) d_{2}^{4}+\frac{1088}{5} \sum_{\substack{x^{2}+y^{2}=2^{\alpha-2} u \\
2 \mid x, x>0, y=0}}\left(x^{4}-3 x^{2} y^{2}\right)+ \\
+\frac{2176}{5} \sum_{2|x, 2| y, x>0, y>0}\left(x^{4}-3 x^{2} y^{2}\right) \text { for } 2 \mid \alpha, \alpha>3, u \equiv 1(\bmod 8), \\
=\frac{4}{5}\left(2^{4 \alpha-1} \cdot 9+1\right) \sum_{d_{1} d_{2}=u}\left(\frac{-1}{d}\right) d_{2}^{4}+\frac{2176}{5} \sum_{\substack{x^{2}+y^{2}=2^{\alpha-2} u \\
2|x, 2| y, x>0, y>0}}\left(x^{4}-3 x^{2} y^{2}\right)
\end{gathered}
$$

for $2 \mid \alpha, \alpha>3, u \equiv 5(\bmod 8)$.

Proof. Equating the coefficients by $Q^{n}$ in both sides of (3.8), we obtain

$$
\begin{aligned}
r(n ; f) & =\rho(n ; f)-\frac{42}{5} \nu_{1}(n)+\frac{544}{5} \nu_{2}(n)- \\
& -\frac{3}{5} \nu_{3}(n)+2 \nu_{4}(n)+24 \nu_{5}(n),
\end{aligned}
$$

where $\nu_{k}(n)(k=1,2,3,4,5)$ denote respectively the coefficients by $Q^{n}$ in the expansions of the functions $\frac{1}{512 \pi^{4}} \Psi_{2}(\tau ; 8,8 ; 0,0 ; 0,0 ; 4,4), \frac{1}{8192 \pi^{4}} \Psi_{2}(\tau$; $0,0 ; 0,0 ; 0,0 ; 4,4), \frac{1}{128 \pi^{4}} \Psi_{2}(\tau ; 4,4 ; 0,0 ; 0,0 ; 2,2),-\frac{1}{32 \pi^{3} i} \Psi_{3}(\tau ; 4,0,4,0 ; 0,1$, $1,1 ; 0,0,0,0 ; 2,4,2,4),-\frac{1}{512 \pi^{3} i} \Psi_{4}(\tau ; 8,8,8,0 ; 1,1,1,1 ; 0,0,0,0 ; 4,4,4,4)$ by powers of $Q$.

From (3.4), (3.11), and (3.12) it follows that

$$
\begin{gathered}
\frac{1}{512 \pi^{4}} \Psi_{2}(\tau ; 8,8 ; 0,0 ; 0,0 ; 4,4)=\sum_{m_{1}, m_{2}=-\infty}^{\infty}\left(2 m_{1}+1\right)^{4} Q^{\left(2 m_{1}+1\right)^{2}+\left(2 m_{2}+1\right)^{2}}- \\
-3 \sum_{m_{1}, m_{2}=-\infty}^{\infty}\left(2 m_{1}+1\right)^{2}\left(2 m_{2}+1\right)^{2} Q^{\left(2 m_{1}+1\right)^{2}+\left(2 m_{2}+1\right)^{2}}
\end{gathered}
$$

i.e.,

$$
\nu_{1}(n)=4 \sum_{\substack{x^{2}+y^{2}=n \\ 2 \dagger x, 2 \dagger y, x>0, y>0}}\left(x^{4}-3 x^{2} y^{2}\right)
$$


it is not difficult to verify that

$$
\nu_{1}(n) \neq 0 \quad \text { for } \alpha=1, \quad u \equiv 1 \quad(\bmod 4) \text { only. }
$$

From (1.16), (3.13), and (3.14) follows

$$
\begin{gathered}
\frac{1}{8192 \pi^{4}} \Psi_{2}(\tau ; 0,0 ; 0,0 ; 0,0 ; 4,4)=\sum_{m_{1}, m_{2}=-\infty}^{\infty} m_{1}^{4} Q^{4 m_{1}+4 m_{2}^{2}}- \\
-3 \sum_{m_{1}, m_{2}=-\infty}^{\infty} m_{1}^{2} m_{2}^{2} Q^{4 m_{1}^{2}+4 m_{2}^{2}}
\end{gathered}
$$

i.e.,

$$
\begin{gathered}
\nu_{2}(n)=4 \sum_{4\left(x^{2}+y^{2}\right)=n}\left(x^{4}-3 x^{2} y^{2}\right)= \\
= \begin{cases}\sum_{x^{2}+y^{2}=2^{\alpha-2} u}\left(x^{4}-3 x^{2} y^{2}\right) & \text { for } \alpha \geq 2, \\
0 & \text { for } \alpha=0,1 ;\end{cases}
\end{gathered}
$$

it is obvious that

$$
\begin{aligned}
\sum_{x^{2}+y^{2}=2^{\alpha-2} u}\left(x^{4}-3 x^{2} y^{2}\right) & =\sum_{\substack{x^{2}+y^{2}=u \\
x \neq y(\bmod 2)}}\left(x^{4}-3 x^{2} y^{2}\right) \text { for } \alpha=2, u \equiv 1(\bmod 4) \\
& =0 \text { for } \alpha \geq 2, u \equiv 3(\bmod 4) \\
& =\sum_{\substack{x^{2}+y^{2}=2^{\alpha-2} u \\
x \equiv y(\bmod 2)}}\left(x^{4}-3 x^{2} y^{2}\right) \text { for } \alpha \geq 3, u \equiv 1(\bmod 4)
\end{aligned}
$$

From (3.5), (3.15), and (3.16) it follows that

$$
\begin{gathered}
\frac{1}{128 \pi^{4}} \Psi_{2}(\tau ; 4,4 ; 0,0 ; 0,0 ; 2,2)=\sum_{m_{1}, m_{2}=-\infty}^{\infty}\left(2 m_{1}+1\right)^{4} Q^{\left(2 m_{1}+1\right)^{2} / 2+\left(2 m_{2}+1\right)^{2} / 2}- \\
-3 \sum_{m_{1}, m_{2}=-\infty}^{\infty}\left(2 m_{1}+1\right)^{2}\left(2 m_{2}+1\right)^{2} Q^{\left(2 m_{1}+1\right)^{2} / 2+\left(2 m_{2}+1\right)^{2} / 2},
\end{gathered}
$$

i.e.,

$$
\begin{aligned}
\nu_{3}(n) & =\sum_{\substack{x^{2}+y^{2}=2 u \\
2 \dagger x, 2 \dagger y}}\left(x^{4}-3 x^{2} y^{2}\right)=4 \sum_{\begin{array}{c}
x^{2}+y^{2}=2 u \\
2 \dagger x, 2 \dagger y, x>0, y>0
\end{array}}\left(x^{4}-3 x^{2} y^{2}\right) \\
& \text { for } \alpha=0, u \equiv 1(\bmod 4), \\
& =0 \quad \text { for } \alpha=0, u \equiv 3(\bmod 4) \text { and for } \alpha>0 .
\end{aligned}
$$


From (3.6) and (3.17)-(3.19) it follows that

$$
\begin{gathered}
-\frac{1}{32 \pi^{3} i} \Psi_{3}(\tau ; 4,0,4,0 ; 0,1,1,1 ; 0,0,0,0 ; 2,4,2,4)= \\
=\left\{\sum_{m_{1}, m_{3}=-\infty}^{\infty}(-1)^{m_{3}}\left(2 m_{1}+1\right)^{2} Q^{\left(2 m_{1}+1\right)^{2} / 2+4 m_{3}^{2}}-\right. \\
\left.\quad-8 \sum_{m_{1}, m_{3}=-\infty}^{\infty}(-1)^{m_{3}} 2 m_{3}^{2} Q^{\left(2 m_{1}+1\right)^{2} / 2+4 m_{3}^{2}}\right\} \times \\
\times \sum_{m_{1}, m_{2}, m_{3}, m_{4}=-\infty}^{\infty}(-1)^{m_{2}+m_{4}}\left(2 m_{2}+1\right) Q^{\left(2 m_{2}+1\right)^{2} / 2+4 m_{4}^{2}}= \\
\quad \times Q^{\frac{1}{2}\left\{\left(2 m_{1}+1\right)^{2}+\left(2 m_{2}+1\right)^{2}+8 m_{3}^{2}+8 m_{4}^{2}\right.},
\end{gathered}
$$

i.e.,

$$
\begin{gathered}
\nu_{4}(n)=\sum_{\substack{x^{2}+y^{2}+8\left(z^{2}+t^{2}\right)=2 n \\
2 \dagger x, 2 \dagger y}}(-1)^{(y-1) / 2+z+t}\left(x^{2}-8 z^{2}\right) y= \\
=4 \sum_{\substack{x^{2}+y^{2}+8\left(z^{2}+t^{2}\right)=2 u \\
2 \dagger x, 2 \dagger y, x>0, y>0}}(-1)^{(y-1) / 2+z+t}\left(x^{2}-8 z^{2}\right) y \\
=0 \quad \text { for } \alpha=0, u \equiv 1(\bmod 4), \\
\\
\quad \text { for } \alpha=0, u \equiv 3(\bmod 4) \text { and for } \alpha>0 .
\end{gathered}
$$

From (3.7) and (3.20) follows

$$
\begin{gathered}
-\frac{1}{512 \pi^{3} i} \Psi_{3}(\tau ; 8,8,8,0 ; 1,1,1,1 ; 0,0,0,0 ; 4,4,4,4)= \\
=\sum_{m_{1}, m_{2}, m_{3}, m_{4}=-\infty}^{\infty}(-1)^{\sum_{k=1}^{4} m_{k}} \prod_{k=1}^{3}\left(2 m_{k}+1\right) Q^{\sum_{k=1}^{3}\left(2 m_{k}+1\right)^{2}+4 m_{4}^{2}},
\end{gathered}
$$

i.e.,

$$
\nu_{5}(n)=8 \sum_{\substack{x^{2}+y^{2}+z^{2}+4 t^{2}=n \\ 2 \dagger x, 2 \dagger y, 2 \dagger z \\ x>0, y>0, z>0}}(-1)^{(x+y+z-3) / 2+t} x y z ;
$$

it is easy to verify that

$$
\nu_{5}(n) \neq 0 \quad \text { only for } \alpha=0, u \equiv 3 \quad(\bmod 4) .
$$


The above theorem follows from (3.26), formulas for $\rho(n ; f)$, and (3.27)(3.31).

\section{REFERENCES}

1. E. Hecke, Analytische Arithmetik der positiven quadratischen Formen. Mathematische Werke, Zweite Auflage, Göttingen, 1970.

2. G. A. Lomadze, On the representation of numbers by some quadratic forms with six variables. (Russian) Trudy Tbilis. Univ., Ser. Mat. Mekh. Astron. 117(1966), 7-43.

3. G. A. Lomadze, On the number of representations of numbers by positive quadratic forms with six variables. (Russian) Trudy Tbilis. Mat. Inst. Razmadze 45(1974), 111-133.

4. G. Lomadze, On some entire modular forms of weights 5 and 6 for the congruence group $\Gamma_{0}(4 N)$. Georgian Math. J. 1(1994), No. 1, 53-75.

5. G. Lomadze, Construction of some modular forms of weights 5 and 6 for the congruence group $\Gamma_{0}(4 N)$. Georgian Math. J. 2(1995), No. 2, 189-199.

6. A. Walfisz, Gitterpunkte in mehrdimensionalen Kugeln. Panstwowe Wydawnictwo Naukowe, Warszawa, 1957.

(Received 01.12.1993)

Author's address:

Faculty of Mechanics and Mathematics

I. Javakhishvili Tbilisi State University

2, University St., Tbilisi 380043

Republic of Georgia 